\title{
High-resolution spectroscopy of RGB stars in the Sagittarius streams
}

\section{Radial velocities and chemical abundances $\star, \star \star$}

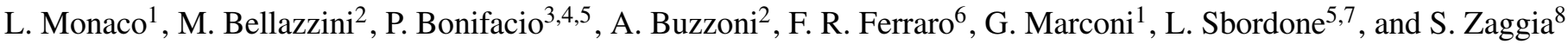 \\ 1 European Southern Observatory, Casilla 19001, Santiago, Chile \\ e-mail: lmonaco@eso.org \\ 2 Istituto Nazionale di Astrofisica - Osservatorio Astronomico di Bologna, 40127 Bologna, Italy \\ CIFIST Marie Curie Excellence Team \\ 4 Observatoire de Paris, GEPI, 5 place Jules Janssen, 92195 Meudon, France \\ 5 Istituto Nazionale di Astrofisica - Osservatorio Astronomico di Trieste, via Tiepolo 11, 34131 Trieste, Italy \\ 6 Università di Bologna - Dipartimento di Astronomia, 40127 Bologna, Italy \\ 7 Istituto Nazionale di Astrofisica - Osservatorio Astronomico di Roma, via Frascati 33, 00040 Monteporzio Catone, Roma, Italy \\ ${ }^{8}$ Istituto Nazionale di Astrofisica - Osservatorio Astronomico di Padova, Vicolo dell’Osservatorio 5, 35122 Padova, Italy
}

Received 11 August 2006 / Accepted 27 October 2006

\section{ABSTRACT}

\begin{abstract}
Context. The Sagittarius (Sgr) dwarf spheroidal galaxy is currently being disrupted under the strain of the Milky Way. A reliable reconstruction of Sgr star formation history can only be obtained by combining core and stream information.

Aims. We present radial velocities for 67 stars belonging to the Sgr Stream. For 12 stars in the sample we also present iron (Fe) and $\alpha$-element (Mg, Ca) abundances.

Methods. Spectra were secured using different high resolution facilities: UVES@VLT, HARPS@3.6 m, and SARG@TNG. Radial velocities are obtained through cross correlation with a template spectra. Concerning chemical analysis, for the various elements, selected line equivalent widths were measured and abundances computed using the WIDTH code and ATLAS model atmospheres. Results. The velocity dispersion of the trailing tail is found to be $\sigma=8.3 \pm 0.9 \mathrm{~km} \mathrm{~s}^{-1}$, i.e., significantly lower than in the core of the Sgr galaxy and marginally lower than previous estimates in the same portion of the stream. Stream stars follow the same trend as Sgr main body stars in the $[\alpha / \mathrm{Fe}]$ vs. $[\mathrm{Fe} / \mathrm{H}]$ plane. However, stars are, on average, more metal poor in the stream than in the main body. This effect is slightly stronger in stars belonging to more ancient wraps of the stream, according to currently accepted models of Sgr disruption.
\end{abstract}

Key words. stars: abundances - stars: atmospheres - galaxies: abundances - galaxies: evolution - galaxies: dwarf galaxies: individual: Sgr dSph

\section{Introduction}

Dwarfs are the most common type of galaxies in the universe. Several dwarf satellites are usually associated with giant galaxies and, in the commonly accepted scenario (White \& Rees 1978), giant galaxies actually emerge out of the hierarchical assembly of dwarfs. In this respect, dwarf galaxies are considered to be the building blocks of the universe.

The Sagittarius dwarf spheroidal galaxy (Ibata et al. 1994, Sgr dSph) is currently disrupting into the Milky Way (MW) and its discovery historically represented one of the first clear confirmations on a local framework of the hierarchical merging paradigm. Nevertheless, the chemical analysis of stars in the

* Based on observations taken at ESO VLT Kueyen telescope (Cerro Paranal, Chile, program: 075.B-0127(A)) and $3.6 \mathrm{~m}$ telescope (La Silla, Chile). Also based on spectroscopic observations taken at the Telescopio Nazionale Galileo, operated by the Fundación G. Galilei of INAF at the Spanish Observatorio del Roque de los Muchachos of the IAC (La Palma, Spain).

$\star \star$ Appendix A and Table 1 are only available in electronic form at http://www. aanda.org
MW satellites and in Sgr itself seems to seriously challenge this evolutionary scheme (see Venn et al. 2004 for a review). In fact, it turned out that red giant stars in local dwarfs have chemical patterns, in particular in the $[\alpha / \mathrm{Fe}]$ abundance ratios, that are not compatible with those of the galactic halo (but see Robertson et al. 2005, for a possible solution to this problem). However, the idea that dwarfs may have contributed the halo with stars even significantly different from their present stellar population is now under investigation (Munoz et al. 2006; Chou et al. 2006).

Tagging accreted components and analyzing their chemical composition is very important for our comprehension of the Milky Way formation. Some streams were identified in the galaxy without any association with a core remnant. Therefore, they could represent the residuals of ancient accretions (Duffau et al. 2006; Belokurov et al. 2006a), and their chemical signature might be very informative as well.

In this framework, Sgr plays a special role. It presents a very significant core remnant ( $30^{\circ}$ tidal radius), and its giant tidal streams (henceforth, the Stream, for brevity), now identified all over the sky (Majewski et al. 2003, hereafter M03), indicate that the disruption process is still ongoing. Hence, Sgr is 
a MW satellite for which a complete reconstruction of the star formation history is possible, combining core and stream information. As such, it will be possible to understand if Sgr is actually a building block of the galactic halo or not. Deep color magnitude diagrams (e.g., Marconi et al. 1998; Bellazzini et al. 1999; Layden \& Sarajedini 2000; Monaco et al. 2002; Bellazzini et al. 2006a) and abundances derived from high resolution spectra (Bonifacio et al. 2000; McWilliam et al. 2003; Bonifacio et al. 2004; McWilliam \& Smecker-Hane 2005; Monaco et al. 2005; Sbordone et al. 2006) provided a fresh wealth of information about the star formation history (SFH) of the stellar populations present in the Sgr core over the years. Information about the Stream is now also accumulating (M03, Majewski et al. 2004; Law et al. 2005; Belokurov et al. 2006b; Chou et al. 2006; Bellazzini et al. 2006b).

This is the first paper of a series devoted to the study of the Sgr Stream. Here, we present radial velocities for 67 red giant branch (RGB) stars belonging to the Stream and high resolution chemical abundances ( $\mathrm{Fe}, \mathrm{Mg}$, and $\mathrm{Ca}$ ) for 12 of these stars. The paper is organized as follows: in Sect. 2 we describe the target selection procedure. The observational dataset and the applied data reduction procedure are discussed in Sect. 3. In Sect. 4 we describe the procedure for radial velocity measurements and discuss the obtained results. In Sect. 5 we present a comparison between radial velocity obtained here and in Majewski et al. (2004, hereafter M04) for a sample of stars belonging to the Sgr trailing tail and common to the two studies. In Sect. 6 we present chemical abundances obtained for 12 stars lying in two different spots of the Stream. In Sect. 7 we discuss our findings. In Sect. 8 we briefly summarize the obtained results.

\section{Target selection}

Data were obtained using three different high resolution facilities. A sub-sample of the M04 stars was observed with UVES (46 stars). The remaining stars were selected from the $2 \mathrm{MASS}^{1}$ catalog employing the M03 procedure, which has already been proven to be a powerful tool to pick-out candidate stream stars (see also M04).

Reddening estimates were obtained through the Schlegel et al. (1998) reddening maps. Distances of the target stars were derived through photometric parallax, following M03, but adopting $(m-M)_{0}=17.10$ as the distance modulus of the Sgr core (Monaco et al. 2004), instead of 16.90. Cartesian coordinates and longitudes in the Sgr orbital plane were derived following M03 (see M03 for definitions and details). Coordinates, magnitudes, and derived distances of the program stars are listed in Table 1. Parameters for UVES stars are taken directly from Table 3 in M04.

In Fig. 1 we plot the position of the target stars (big solid markers) in Cartesian coordinates of the (galactocentric) Sgr orbital plane. Different symbols correspond to stars observed with different facilities. The Law et al. (2005) model of Sgr destruction (for a spherical galactic potential) is also plotted for reference.

\section{Observations and data reduction}

A total of 13 stars were observed between August 30, 2004, and January 24, 2005, using the SARG spectrograph mounted on the Telescopio Nazionale Galileo (TNG) telescope at La Palma. We used the 1". 6 slit, which provides a resolution of $R=29000$.

\footnotetext{
${ }^{1}$ See http://www.ipac.caltech.edu/2mass
}

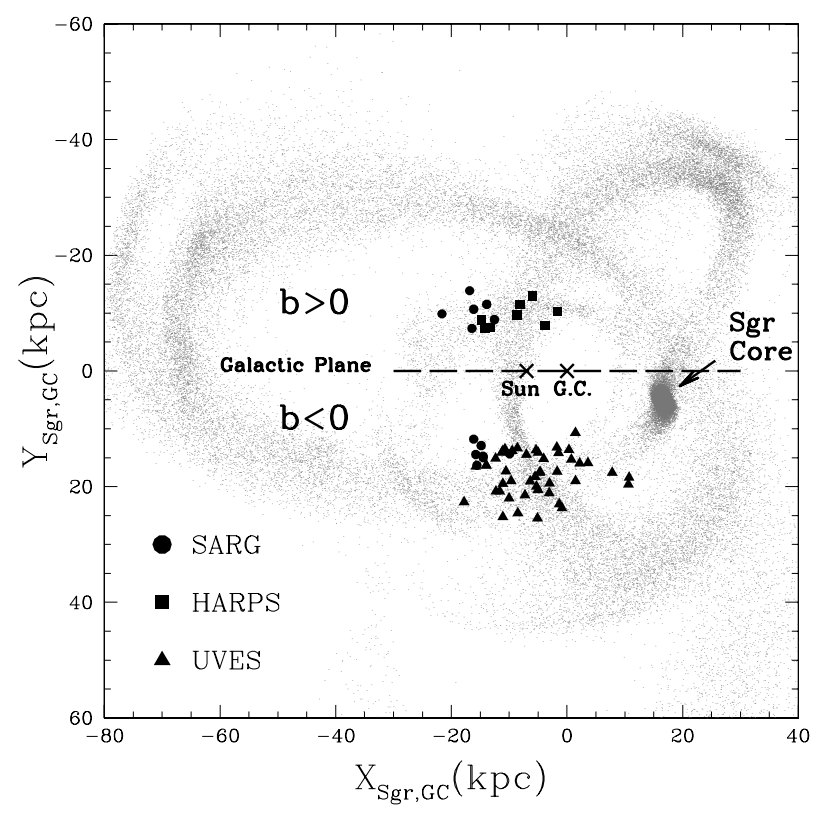

Fig. 1. Program stars positions in the Cartesian galactocentric Sgr plane (filled symbols). Different symbols correspond to stars observed with different spectrographs. A model of the Sgr disruption (grey dots) is also plotted. The galactic plane (dashed line) and the position of the Sun, of the galactic center, and of the Sgr main body are also marked for reference.

The chosen setup used the yellow cross-disperser, which covers approximately the $462-792 \mathrm{~nm}$ spectral range. Data reduction (bias subtraction, division by flat field, lambda calibration, background subtraction, and extraction) was performed within the ESO-MIDAS ${ }^{2}$ echelle context.

During a technical-time slot on the nights of June 3 and 4 , 2006, we observed 8 supplementary stars with the HARPS facility mounted at the $3.6 \mathrm{~m}$ telescope in La Silla. The standard high resolution HARPS mode ( $R=110000,380-690 \mathrm{~nm}$ spectral range) was employed. Stars were observed for an integration time ranging from $800 \mathrm{~s}$ (\#465) to $1200 \mathrm{~s}$ (all the others). Additional HARPS observations were obtained for stars \#1006, \#1022, and \#1083 in July 2006, with 30 min exposures. The June 29, 2006 star \#1006 was observed for one hour integration time.

Data were reduced through the online automatic pipeline installed on the WHALDRS workstation at the $3.6 \mathrm{~m}$ control room. The final output of the HARPS pipeline is extracted spectra that are completely reduced (bias-subtraction, cosmic rays filtering, flat-field, and wavelength calibration), and the star radial velocity as measured by a cross correlation function on the bidimensional echelle spectrum with a template G2 dwarf ${ }^{3}$ mask. The extreme stability of the HARPS facility secures accurate radial velocity measures even with very low signal-to-noise spectra. In Fig. 2 we plot the cross correlation function obtained for the two lowest $S / N$ spectra obtained. The signal corresponding to the star radial velocity is clearly evident.

${ }^{2}$ ESO-MIDAS is the acronym for the European Southern Observatory Munich Image Data Analysis System, developed and maintained by the European Southern Observatory. http://www.eso.org/projects/esomidas/

3 To date, the G2 dwarf is the only template available for cross correlation using the HARPS pipeline. However, using a not yet released M4 mask, we found a $\sim 100 \mathrm{~m} \mathrm{~s}^{-1}$ radial velocity difference in a test made on star \#459992. 


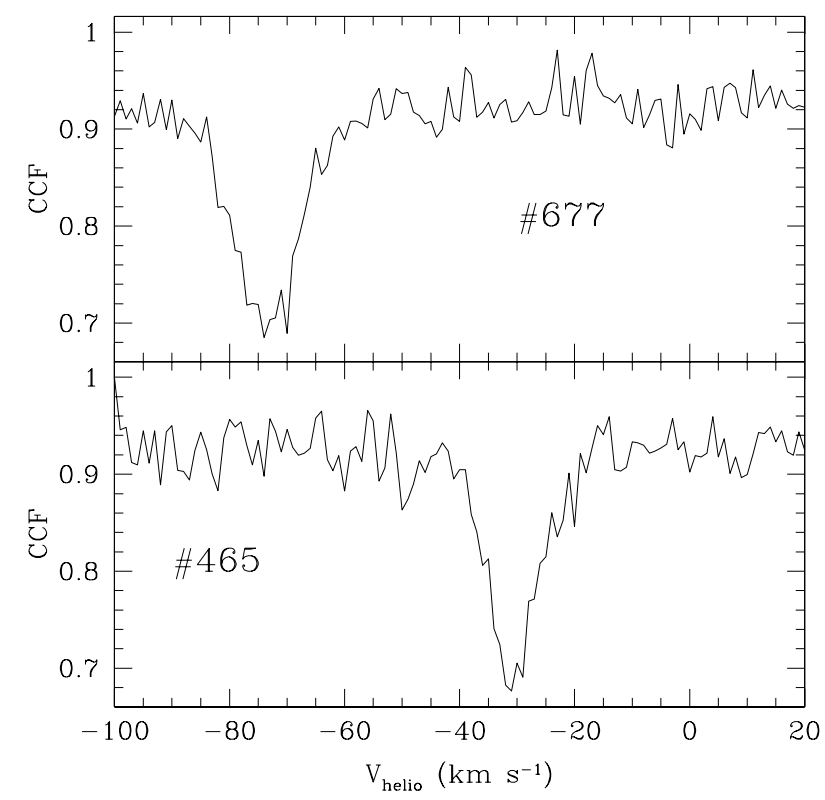

Fig. 2. Cross correlation function (CCF) of the two lowest signal-tonoise HARPS spectra with the G2 dwarf template. The heliocentric star radial velocity reported in Table 1 is obtained through a Gaussian fit to the observed peak. Contrary to usual conventions, the star radial velocity is found by the HARPS pipeline as a minimum in the CCF.

UVES spectra for 46 stars were obtained between June 18 and September 16, 2005. Stars were observed with the standard setting DIC $390+580 \mathrm{~nm}$, which covers the spectral range 328-456 nm and 480-680 nm, with the Blue and Red arms, respectively. We employed a $2 \times 2 \mathrm{CCD}$ binning and a slit width of 1 '.2, which provide a resolution of about $35000 \div 40000$. Data were reduced using the UVES ESO-MIDAS pipeline.

\section{Radial velocities}

Radial velocities (RV) of star in the SARG and UVES samples are obtained by cross correlation with a synthetic spectrum using the fxcor task inside the IRAF ${ }^{4}$ suite. The synthetic spectrum was calculated employing the SYNTHE code (Kurucz 1993a; Sbordone et al. 2004) and a set of atmospheric parameters (temperature; gravity; metallicity $=3900 ; 1.0 ;-0.5)$ similar to those of all the observed stars (see, e.g., Table 3). Concerning the HARPS spectra, the formal photon noise induced radial velocity error is in the worst case $11 \mathrm{~m} \mathrm{~s}^{-1}$. A conservative $200 \mathrm{~m} \mathrm{~s}^{-1}$ uncertainty is assumed. In Table 1 we report the measured radial velocities (heliocentric and in the galactic standard of $\operatorname{rest}^{5}$ ), as well as the signal-to-noise ratio of the spectra. Radial velocities are obtained with a precision, generally, better than $0.5 \mathrm{~km} \mathrm{~s}^{-1}$. For the HARPS and UVES spectra, we only present radial velocities, here.

In Fig. 3 we plot the program stars RVs (in the galactic standard of rest, $\left.v_{\text {gsr }}\right)$ as a function of the Sgr longitude scale $\left(\Lambda_{\odot}\right)$ along the orbital plane. We also plot M04 (for distances larger than $13 \mathrm{kpc}$ ) and Dohm-Palmer et al. (2001) data superposed to

${ }^{4}$ IRAF is distributed by the National Optical Astronomy Observatories, which is operated by the association of Universities for Research in Astronomy, Inc., under contract with the National Science Foundation.

5 A local standard of rest rotation velocity of $220 \mathrm{~km} \mathrm{~s}^{-1}$ and a peculiar motion of $(u, v, w)=(-9,12,7) \mathrm{km} \mathrm{s}^{-1}$ are adopted for the Sun, for consistency with M04.

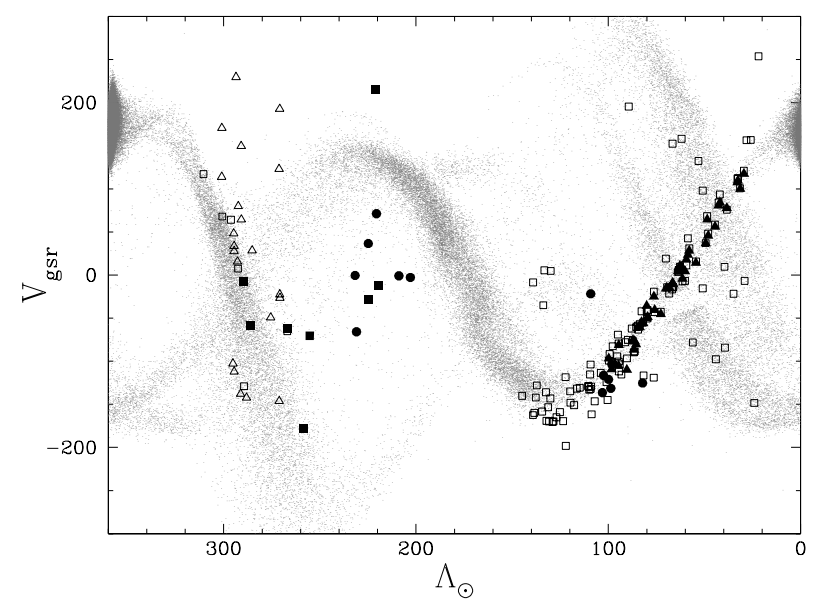

Fig. 3. Galactic standard of rest radial velocities of the program stars (filled symbols) as a function of the longitude of the Sgr orbital plane. Different symbols correspond to stars observed with different spectrographs (circles, squares, and triangles for SARG, HARPS, and UVES data, respectively). Stars studied by M04 (empty squares) and by Dohm-Palmer et al. (2001) (open triangles) are also plotted together with a model of the Sgr disruption (grey dots).

the Sgr destruction model already used in Fig. 1. Stars in the UVES sample describe a characteristic trend of decreasing $v_{\text {gsr }}$ with increasing $\Lambda_{\odot}$ along the Sgr trailing tail, as already discussed by M04. The same trend is also followed by SARG stars at similar stream positions. Referring to Fig. 1, at positive galactic latitude $\left(b>0\right.$ or $\left.Y_{\mathrm{Sgr}, \mathrm{GC}}<0\right)$ all the SARG and 3 among the HARPS stars lie on a well defined branch of stream $\left(X_{\mathrm{Sgr}, \mathrm{GC}}<\right.$ $-10 \mathrm{kpc})$. The large dispersion shown by this group in Fig. 3 $\left(\Lambda_{\odot}<230^{\circ}\right)$ is predicted to some extent by the model, and more data are mandatory to constrain the radial velocity pattern of this part of the stream. The remaining part of the HARPS stars lie in a region where different branches of the Sgr Stream overlap $\left(X_{\mathrm{Sgr}, \mathrm{GC}}>-10 \mathrm{kpc}\right.$ in Fig. 1$)$. Their radial velocities nicely fit with the trend predicted by the model for the Sgr leading tail and confirmed by Dohm-Palmer et al. (2001) (Fig. 3) and Law et al. (2005) data. However, especially for the three stars at $230^{\circ}<\Lambda_{\odot}<280^{\circ}$, some ambiguity still holds.

\section{The UVES sample: comparison with Majewski et al. (2004)}

\subsection{Sanity check and possible binary stars}

UVES stars were selected out of the M04 sample and trace 70 degrees of the Sgr trailing tail, in the range $30^{\circ}<\Lambda_{\odot}<$ $100^{\circ}$. Note that $\Lambda_{\odot}=0$ at the Sgr core. Figure 4 shows the distribution of the differences between the heliocentric radial velocity measured here and in M04 (see Table 1). After removing star \#1006 (which shows a remarkably large velocity difference: $-35.4 \mathrm{~km} \mathrm{~s}^{-1}$ ), the distribution is well represented by a Gaussian distribution centered at $-0.44 \mathrm{~km} \mathrm{~s}^{-1}$ and having a $\sigma$ of $5.45 \mathrm{~km} \mathrm{~s}^{-1}$. Hence, there is no zero point difference between the two sets of measures and, given the high accuracy of the UVES velocities, the dispersion of the distribution nicely confirms the $5.3 \mathrm{~km} \mathrm{~s}^{-1}$ quoted by M04 as random errors.

It is noteworthy that 3 stars lie over the $3 \sigma$ limit $\left(>16 \mathrm{~km} \mathrm{~s}^{-1}\right.$ of RV variation, Fig. 4). A possible reason for the detected RV difference is that these stars are in fact binary systems, observed at different orbital phases. Time series of RV measures are clearly needed to assess this hypothesis on a firm basis. Between 


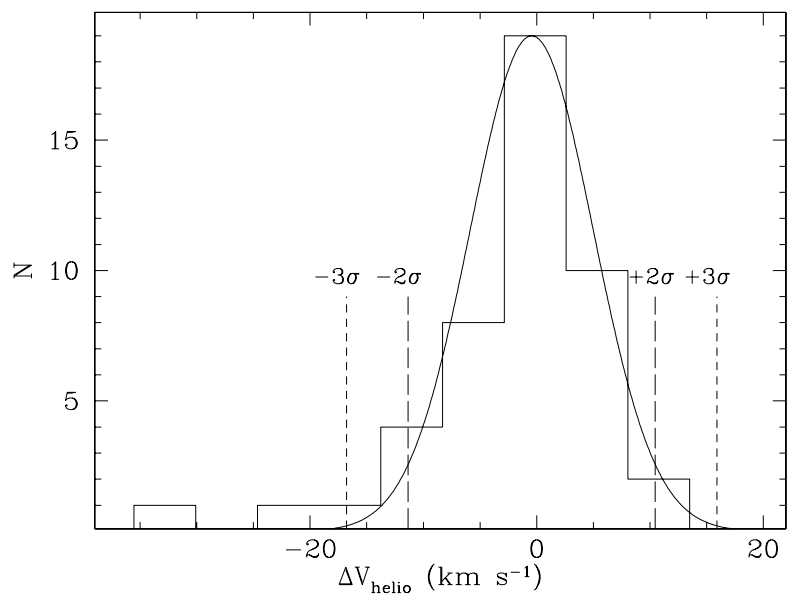

Fig. 4. Distribution of the difference between RVs measured here and in M04. The best-fit Gaussian curve $\left(\sigma=5.45 \mathrm{~km} \mathrm{~s}^{-1}\right)$ is also plotted. Long dashed and short dashed lines mark $2 \sigma$ and $3 \sigma$ levels.

Table 2. Heliocentric radial velocity at different dates for the three suspected binaries. Brackets besides dates acknowledge UVES (U), HARPS (H), or M04 measures. The last column reports the signal-tonoise ratio of the HARPS and UVES spectra or the cross-correlation quality index for M04 data.

\begin{tabular}{ccccc}
\hline \hline & & $v\left(\mathrm{~km} \mathrm{~s}^{-1}\right)$ & & \\
Date & 1006 & 1022 & 1083 & $\mathrm{Q}$ \\
\hline $2002-07-15$ (M04) & & & -0.1 & 7 \\
2002-07-30 (M04) & & -82.8 & & 5 \\
2002-07-31 (M04) & -6.5 & & & 7 \\
\hline & & & & $S / N$ \\
\hline $2005-07-19(\mathrm{U})$ & & & -19.21 & 14 \\
$2005-07-20(\mathrm{U})$ & -41.90 & & & 42 \\
$2005-09-13(\mathrm{U})$ & & -102.48 & & 18 \\
$2006-06-29(\mathrm{H})$ & -46.65 & & & 10 \\
$2006-07-14(\mathrm{H})$ & & -105.14 & & 9 \\
$2006-07-15(\mathrm{H})$ & & & -19.71 & 8 \\
$2006-07-17(\mathrm{H})$ & -47.33 & & & 13 \\
$2006-07-19(\mathrm{H})$ & -47.25 & -104.64 & & 12,11 \\
\hline
\end{tabular}

June and July 2006, additional HARPS data was obtained for these stars. In Table 2 we report a summary of the RVs measured for stars \#1006, \#1022, and \#1083. Support of the binary hypothesis is provided by this new data to star \#1006 and, to some extent, also to \#1022, while no significant RV variation between the UVES and HARPS measures was obtained for star \#1083. In any case, considering the 3 outliers as genuine binaries, a preliminary lower limit for the Sgr binary fraction of $\sim 6 \%$ is derived.

\subsection{The velocity dispersion of the Sgr trailing tail}

In the upper panels of Fig. 5, we plot the $v_{\text {gsr }}$ as a function of $\Lambda_{\odot}$ for stars in the UVES sample. The left panel shows our measures, and the right panel M04 RVs. Continuous lines show a least-squares fit and a polynomial fit (M04) to the trend, in the former and latter cases, respectively. The fits hold up to $\Lambda_{\odot}<$ $90^{\circ}$, where the increase of the velocity dispersion is evident (see M04).

Lower panels show the distribution of differences between the actual RV and the fit. M04 data (right panel) is well fitted by a Gaussian curve having a dispersion of $11.8 \mathrm{~km} \mathrm{~s}^{-1}$, once star \#1006 (which lies more than $3 \sigma$ away from the mean) is removed. M04 used a $\sigma=11.7 \mathrm{~km} \mathrm{~s}^{-1}$ Gaussian curve to fit the
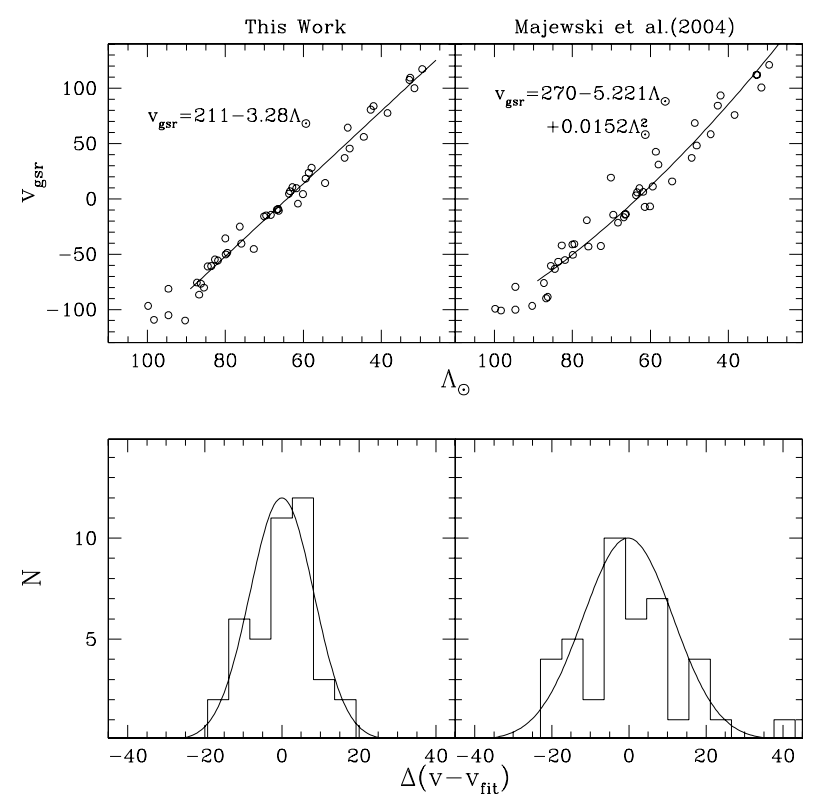

Fig. 5. Upper panels: measured radial velocities as a function of the longitude of the Sgr orbital plane for stars in the UVES sample. The left panel shows measures presented here; the right panel shows M04 measures. The fit to the observed distributions are also plotted. Lower panels: distribution of the differences between the fit and the actual RV for the two set of measures.

observed distribution. Hence, the stars we observed are representative of the more populous M04 sample. Note also that M04 used 45 stars to evaluate the stream velocity dispersion, a number not so different from the 40 objects we use here.

The left lower panel shows residuals of our measures with respect to the fit. The distribution is fitted by a Gaussian of $\sigma=8.3 \pm 0.9 \mathrm{~km} \mathrm{~s}^{-1}$ (without rejecting any star, i.e., using 41 stars) while M04 obtained an intrinsic stream dispersion of $\sigma=10.4 \pm 1.3 \mathrm{~km} \mathrm{~s}^{-1}$, after removing the random errors $\left(\sim 5.3 \mathrm{~km} \mathrm{~s}^{-1}\right)$. The two values are in agreement, within the errors. However, the above results suggest that this part of the trailing tail is colder than what was estimated by M04 with low resolution spectroscopy, and it also appears colder than the Sgr core $\left(11.17 \mathrm{~km} \mathrm{~s}^{-1}\right.$ and $11.4 \mathrm{~km} \mathrm{~s}^{-1}$ in Monaco et al. 2005, hereafter M05; and Ibata et al. 1997, respectively). Nonetheless, the external regions of the Sgr main body may present velocity dispersions more similar to what observed in this portion of the Stream (see also Ibata et al. 1997).

Our results suggest that to properly characterize dynamical structures these cold (e.g., streams in and outside the halo, dwarf galaxies velocity dispersions), high resolution data are really useful, if not mandatory. It should be also kept in mind that a sizable population of binaries could (and indeed should) be present in Sgr. However, the increase of the measured velocity dispersions of a dwarf galaxy due to the presence of a binary population should be considered at most marginal (Hargreaves et al. 1996; Olszewski et al. 1996).

At $\Lambda_{\odot}>90$, we confirm the M04 claim of a rise in the stream velocity dispersion. However, with just 5 stream stars no meaningful comparison with M04 can be done. Note also that a colder velocity dispersion in the stream of a disrupting system with respect to the core remnant is expected on the basis of the conservation of phase-space density (see Helmi \& White 1999). The stream velocity dispersion should actually decrease as a function of time (as $\frac{1}{t}$ ). 


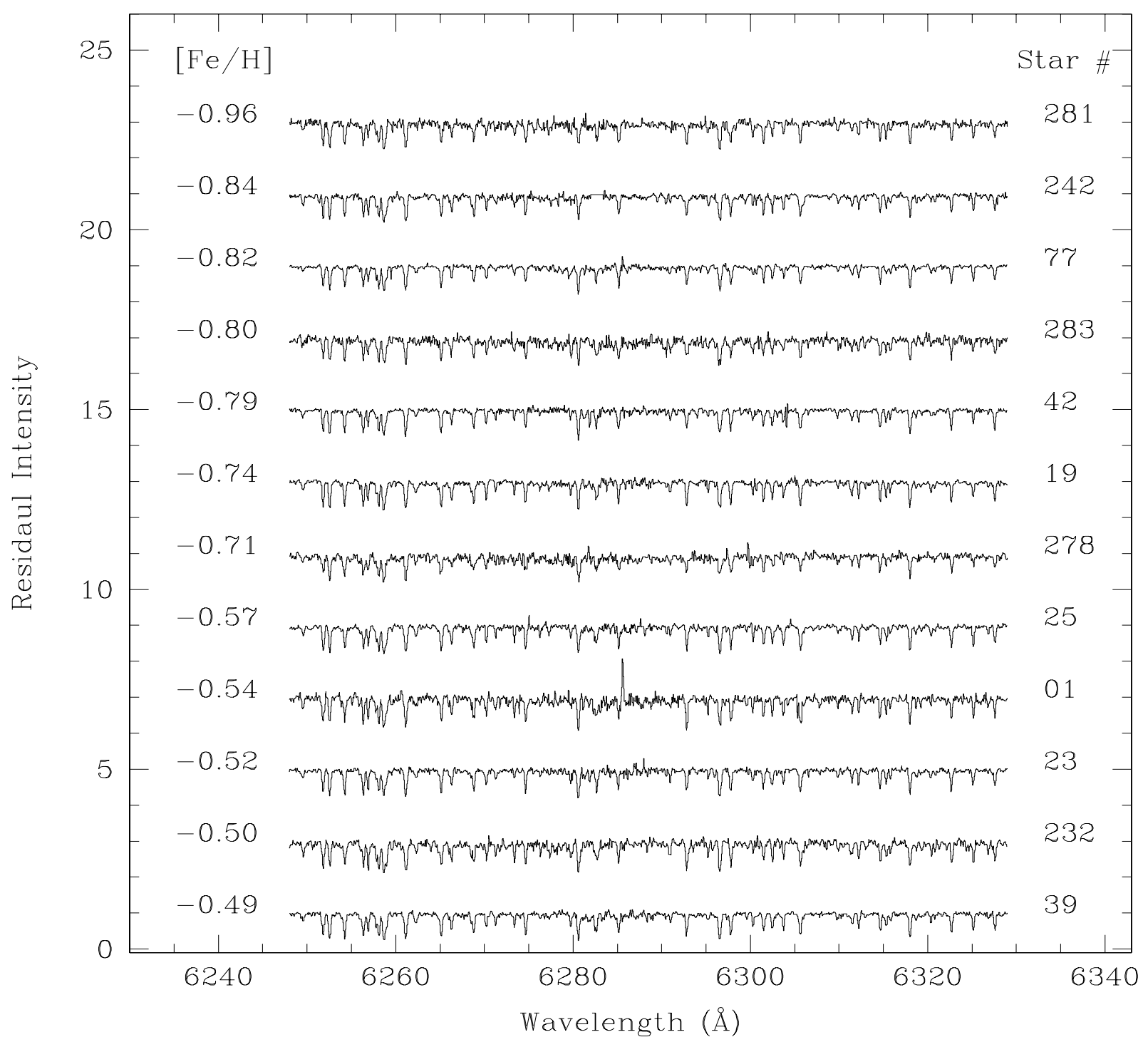

Fig. 6. Sample of the SARG spectra of the 12 stream stars for which the chemical analysis was performed. Labels on the right denote the star number, those on the left the measured $[\mathrm{Fe} / \mathrm{H}]$.

\section{Chemical analysis}

Looking at Fig. 1, it is easy to realize that SARG stars lie at the most extreme Stream positions, among the program stars. They sample two different wraps of Stream and in each of them a part of the Stream significantly distant from the Sgr core. Therefore, SARG stars are ideal to spot the basic chemical characteristics of the Sgr Stream. Here we present $\mathrm{Fe}, \mathrm{Mg}$, and $\mathrm{Ca}$ abundances for the stars in the SARG sample.

In more detail, stars from \#1 to \#77 of Table 1 belong to the trailing tail $\left(b<0\right.$ or $Y_{\mathrm{GC}, \mathrm{Sgr}}>0$, Fig. 1) at more than 80 degrees from the Sgr core. Stars from \#232 to \#283 ( $b>0$ or $Y_{\mathrm{GC}, \mathrm{Sgr}}<$ 0 ), lie above the galactic plane and probably belong to a more ancient branch of the stream. Star \#260 has a too low $S / N$ ratio (see Table 1) to allow a reliable chemical analysis and is, therefore, dropped in the following discussion. In Fig. 6 we plot a sample of the SARG spectra of the 12 stars for which the chemical analysis was performed.

Stars in the UVES sample have cooler temperatures with respect to SARG stars. As such, the great majority of them present deep titanium oxide bands (TiO, see Fig. 4 in M05), which strongly complicates the chemical analysis. TiO bands depress the continuum, and a reliable estimate of the continuum level is crucial for robust equivalent width measurements. Thus, the derivation of elemental abundances for such cool stars represents a significant challenge. A few groups are actively investigating methods to derive trustworthy abundances for $\mathrm{M}$ stars by the simultaneous comprehensive synthesis of selected spectral regions roughly in the range $7000 \AA<\lambda<9000 \AA$ (see Valenti et al. 1998; Bean et al. 2006). For this reason and in spite of the high quality of the data, the analysis of stars in the UVES sample will be presented in a forthcoming contribution.

\subsection{Atmospheric parameters and chemical abundances}

Dereddened $(J-K)$ colors were used together with the Alonso et al. (1999) calibration to derive the effective temperature $\left(T_{\text {eff }}\right)$ of the program stars. Stars share very similar colors $\left((J-K)_{0}=\right.$ $0.93 \div 0.97$, see Table 1$)$, which turn into a quite tight range of temperature, namely $T_{\text {eff }}=3831 \div 3936 \mathrm{~K}$. Note that effective temperatures derived with this procedure, however, appear on average roughly $2 \%$ hotter (i.e., $+76 \mathrm{~K}$ ) than the calibration scale 
Table 3. Atmospheric parameters assumed for the program stars.

\begin{tabular}{lrrrr}
\hline \hline Star & $T_{\text {eff }}$ & $\log g$ & $\xi$ & {$[\mathrm{M} / \mathrm{H}]$} \\
\hline 1 & 3900 & 0.9 & 2.1 & -0.5 \\
19 & 3900 & 0.9 & 2.1 & -0.5 \\
23 & 3900 & 0.9 & 2.0 & -0.5 \\
25 & 3900 & 0.9 & 2.0 & -0.5 \\
39 & 3900 & 0.9 & 1.7 & -0.5 \\
42 & 3900 & 0.9 & 2.1 & -0.5 \\
77 & 3900 & 0.9 & 1.9 & -0.5 \\
232 & 3900 & 0.9 & 2.1 & -0.5 \\
242 & 3900 & 0.9 & 2.2 & -0.5 \\
278 & 3900 & 0.9 & 2.0 & -0.5 \\
281 & 3900 & 0.9 & 2.2 & -0.5 \\
283 & 3900 & 0.9 & 2.0 & -0.5 \\
\hline
\end{tabular}

Table 4. Mean chemical abundances of the program stars. The number of lines used and the line scatter are also reported.

\begin{tabular}{lllllll}
\hline \hline Star & $A(\mathrm{Fe})$ & $n$ & $A(\mathrm{Mg})$ & $n$ & $A(\mathrm{Ca})$ & $n$ \\
\hline Sun & 7.51 & & 7.58 & & 6.35 & \\
1 & $6.96 \pm 0.28$ & 28 & $7.08 \pm 0.19$ & 4 & $5.57 \pm 0.20$ & 7 \\
19 & $6.77 \pm 0.22$ & 36 & $6.80 \pm 0.25$ & 4 & $5.38 \pm 0.19$ & 8 \\
23 & $7.00 \pm 0.27$ & 33 & $6.83 \pm 0.12$ & 2 & $5.51 \pm 0.14$ & 7 \\
25 & $6.94 \pm 0.22$ & 31 & $7.15 \pm 0.25$ & 4 & $5.52 \pm 0.14$ & 7 \\
39 & $7.02 \pm 0.22$ & 33 & $6.80 \pm 0.14$ & 3 & $5.56 \pm 0.17$ & 7 \\
42 & $6.70 \pm 0.22$ & 32 & $6.93 \pm 0.09$ & 3 & $5.41 \pm 0.25$ & 8 \\
77 & $6.69 \pm 0.25$ & 32 & $7.00 \pm 0.06$ & 4 & $5.63 \pm 0.27$ & 6 \\
232 & $7.01 \pm 0.20$ & 25 & $7.51 \pm 0.20$ & 4 & $5.94 \pm 0.23$ & 7 \\
242 & $6.67 \pm 0.29$ & 27 & $7.05 \pm 0.11$ & 3 & $5.49 \pm 0.15$ & 7 \\
278 & $6.80 \pm 0.24$ & 17 & $6.89 \pm 0.14$ & 3 & $5.54 \pm 0.16$ & 7 \\
281 & $6.54 \pm 0.18$ & 25 & $6.83 \pm 0.19$ & 4 & $5.14 \pm 0.17$ & 7 \\
283 & $6.71 \pm 0.20$ & 26 & $6.94 \pm 0.15$ & 4 & $5.41 \pm 0.23$ & 5 \\
\hline
\end{tabular}

$A(\mathrm{X})=\log \left(\frac{X}{H}\right)+12.00$.

adopted in $\mathrm{M}^{6} 5^{6}$. We eventually adopted $T_{\text {eff }}=3900 \mathrm{~K}$ for all of our stars, assuming a $\pm 100 \mathrm{~K}$ uncertainty. However, note that (i) the assumed $T_{\text {eff }}$ obtain excitation equilibrium of the neutral iron lines (Fe I) in all but two (\#242 and \#42) of the stream stars, and that (ii) $76 \mathrm{~K}$ of difference in the temperature scale do not induce any sensible change in the derived abundances, as can be seen from Table 6 and Table 4 in M05.

All the targets were photometrically classified as M-Giants (see M03). However, photometric classification is always tentative and should be spectroscopically confirmed. Stars are classified as M-type on the basis of the presence of titanium oxide $(\mathrm{TiO})$ bands in their spectra. Indeed, the SARG spectra do not present $\mathrm{TiO}$ bands, as somewhat expected from their not exceedingly low temperatures (see also M05). Thus, chemical abundances are safely derived from spectral lines equivalent widths $(E W)$ provided a proper model atmosphere is employed.

After correcting for their distance and reddening, gravity should be derived for target stars by the relevant fundamental relationship:

$\log g=4 \log T_{\text {eff }}-\log L_{*}+\log M_{*}+$ const,

where const $=\log (4 \pi G \sigma)=-10.32$ and $M_{*}$ and $L_{*}$ are the stellar mass and luminosity. However, given the obvious uncertainty in the definition of both the stellar mass and the bolometric correction at such low temperatures, only a safe physical range can

${ }^{6}$ In M05, $T_{\text {eff }}$ were derived for stars in the Sgr core from optical colors. The quoted $2 \%$ of difference in the temperature scale was estimated comparing M05 stars temperatures as derived from optical and infrared colors.
Table 5. Mean abundance ratios for the program star. For iron abundances, the line scatter is also reported.

\begin{tabular}{lcrr}
\hline \hline Star & {$[\mathrm{Fe} / \mathrm{H}]$} & {$[\mathrm{Mg} / \mathrm{Fe}]$} & {$[\mathrm{Ca} / \mathrm{Fe}]$} \\
\hline 1 & $-0.55 \pm 0.28$ & 0.05 & -0.23 \\
19 & $-0.74 \pm 0.22$ & -0.04 & -0.23 \\
23 & $-0.51 \pm 0.27$ & -0.24 & -0.33 \\
25 & $-0.57 \pm 0.22$ & 0.14 & -0.26 \\
39 & $-0.49 \pm 0.22$ & -0.29 & -0.30 \\
42 & $-0.81 \pm 0.22$ & 0.16 & -0.13 \\
77 & $-0.82 \pm 0.25$ & 0.24 & 0.10 \\
232 & $-0.50 \pm 0.20$ & 0.43 & 0.09 \\
242 & $-0.84 \pm 0.29$ & 0.31 & -0.02 \\
278 & $-0.71 \pm 0.24$ & 0.02 & -0.10 \\
281 & $-0.97 \pm 0.18$ & 0.22 & -0.24 \\
283 & $-0.80 \pm 0.20$ & 0.16 & -0.14 \\
\hline
\end{tabular}

$[\mathrm{X} / \mathrm{Y}]=\log \left(\frac{X}{Y}\right)-\log \left(\frac{X}{Y}\right)_{\odot}$

Table 6. Errors in the abundances of star \#19 due to uncertainties in the atmospheric parameters.

\begin{tabular}{lrrr}
\hline \hline & $\Delta A(\mathrm{Fe})$ & $\Delta A(\mathrm{Mg})$ & $\Delta A(\mathrm{Ca})$ \\
\hline$\Delta \xi= \pm 0.2 \mathrm{~km} \mathrm{~s}^{-1}$ & -0.10 & $\mp 0.04$ & -0.11 \\
& +0.12 & & +0.12 \\
$\Delta T_{\text {eff }}= \pm 100 \mathrm{~K}$ & -0.01 & -0.01 & +0.10 \\
& +0.04 & +0.04 & -0.09 \\
$\Delta \log g= \pm 0.50$ & +0.15 & +0.08 & -0.01 \\
\hline
\end{tabular}

be identified for $\log g$, relying on a collection of isochrones (see, e.g., Fig. 11 in Bertone et al. 2004). Comparing with the Girardi et al. (2002) isochrones in the K vs. $(J-K)$ plane, we derived $\log g=0.9 \pm 0.5$ dex as a realistic estimate of the representative surface gravity and its allowed range, for all the targets.

To derive the chemical abundances, we firstly calculated a model atmosphere with $T_{\text {eff }}=3900 \mathrm{~K}, \log g=0.9,[\mathrm{M} / \mathrm{H}]=$ -0.5 , and the Opacity Distribution Functions of Kurucz (1993b). Secondly, we measured $E W \mathrm{~s}$ on the spectra for a selected sample of $\mathrm{Fe}, \mathrm{Mg}$, and $\mathrm{Ca}$ lines using the standard IRAF task splot. Finally, abundances were derived from the measured $E W$ s using the calculated model atmosphere within the WIDTH code. The GNU-Linux ported version (Sbordone et al. 2004) of both the WIDTH and ATLAS codes (Kurucz 1993a) were employed. Microturbulent velocities $(\xi)$ for each star were determined minimizing the dependence of the iron abundance from the $E W$.

The atmospheric parameters adopted for the program stars are reported in Table 3 . The $\mathrm{Fe}, \mathrm{Ca}$, and $\mathrm{Mg}$ line lists, as well as the adopted atomic parameters and the measured $E W$, are reported in Table A.1. Table A.1 also lists the abundance obtained for each line. The mean and standard deviation of such abundances can be found in Tables 4 and 5 (as $[\mathrm{X} / \mathrm{H}]$ abundances in the latter case) for each chemical species together with the number of lines employed. The line scatter reported in Tables 4 and 5 should be representative of the statistical error arising from the noise in the spectra and from uncertainties in the measurement of the equivalent widths ${ }^{7}$. In Table 6 we report the errors arising from the uncertainties in the atmospheric parameters in the case of star \#19, taken as representative of the whole sample.

\footnotetext{
7 Under the assumption that each line provides an independent measure of the abundance, the error in the mean abundances should be obtained by dividing the line scatter by $\sqrt{n}$ (where $n$ is the number of measured lines). However, we consider the line scatter reported in Tables 4 and 5 (which is not divided by $\sqrt{n}$ ) as a realistic estimate of the error associated with each abundance.
} 


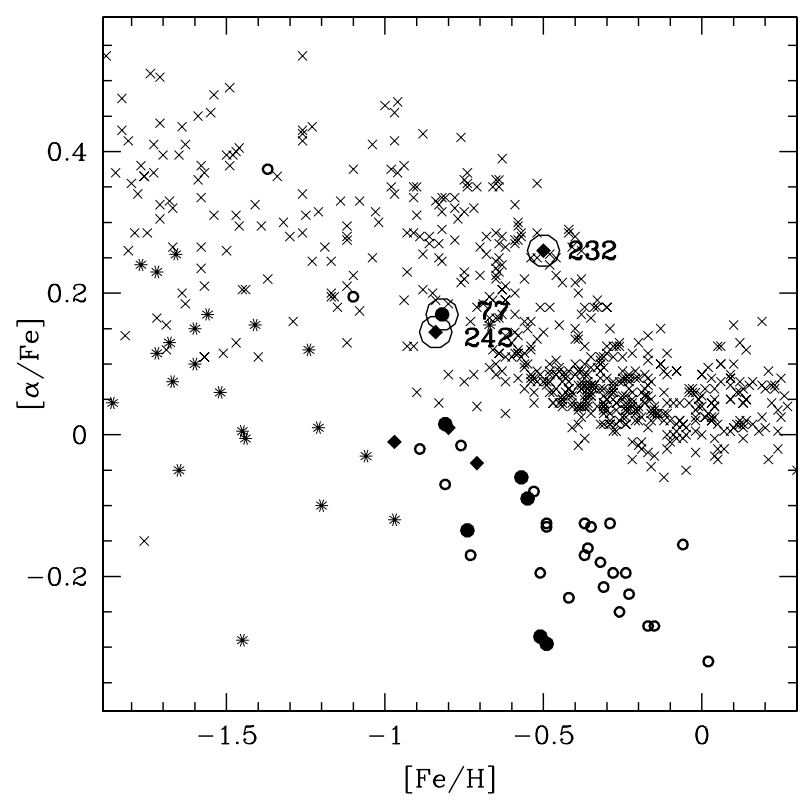

Fig. 7. $[\alpha / \mathrm{Fe}]=\frac{[\mathrm{Ca} / \mathrm{Fe}]+[\mathrm{Mg} / \mathrm{Fe}]}{2}$ as a function of the $[\mathrm{Fe} / \mathrm{H}]$ for MW stars and local group galaxies (crosses and asterisks, respectively, from Venn et al. 2004). Filled symbols refer to program stars (diamonds for the $b>$ 0 subsample) while Sgr main body stars are plotted as empty circles.

In Fig. 7 we plot the mean alpha element abundance ratio (defined as $\langle[\alpha / \mathrm{Fe}]\rangle=\frac{[\mathrm{Mg} / \mathrm{Fe}]+[\mathrm{Ca} / \mathrm{Fe}]}{2}$ as in M05) as a function of the measured $[\mathrm{Fe} / \mathrm{H}]$. Chemical abundances of main body stars from $\mathrm{M} 05^{8}$ and $\mathrm{B} 04^{9}$ are plotted. MW and Local group dwarfs stars are plotted as well (Venn et al. 2004). Stream stars, clearly, follow the same trend defined by the stars that are still bound to the core of Sgr. Stars belonging to the $b>0$ subsample are indicated.

Stars \#77, \#232, and \#242 occupy a portion of plane dominated by MW stars in Fig. 7. Unlike \#232, stars \#77 and \#242 lie in a transition region where their abundances are still compatible with the Sgr path. Star \#77 also has a relatively high RV $\left(v_{\mathrm{gsr}}=-21.1\right)$ compared with the mean stream pattern $\left(\Lambda_{\odot}=\right.$ 109.2, see Fig. 3 and Table 1). On the other hand, \#242 RV is similar to other SARG and HARPS stars lying at similar stream longitudes (Fig. 3). Hence, in the following analysis we conservatively drop \#77 and \#232 as possible contaminating MW stars. We keep star \#1, in spite of its slightly low RV, since its chemical composition follows the Sgr pattern. The inclusion or exclusion of this star does not substantially modify our conclusions.

\section{Discussion}

We presented RV for a sample of 67 stars belonging to the Sgr Stream. Spectra were obtained using 3 different high resolution facilities, namely SARG@TNG, HARPS@3.6 m, and UVES@VLT. Stars in the UVES sample (46 stars) trace $70^{\circ}$ along the trailing tail and were already observed at low resolution by M04. We found a trailing tail velocity dispersion of $8.3 \pm$ $0.9 \mathrm{~km} \mathrm{~s}^{-1}$, a value in marginal agreement with M04 (10.4 \pm $1.3 \mathrm{~km} \mathrm{~s}^{-1}$ ) and colder than the Sgr core (Ibata et al. 1997, M05).

\footnotetext{
${ }^{8}$ Only the 15 stars not showing TiO bands are plotted. See Table 1 in M05.

9 The B04 abundances were recomputed adopting the same temperature scale and reddening adopted in M05. These variations in the input parameters produced small (compatible with the quoted errors) changes in the derived abundances (see Sbordone et al. 2006).
}
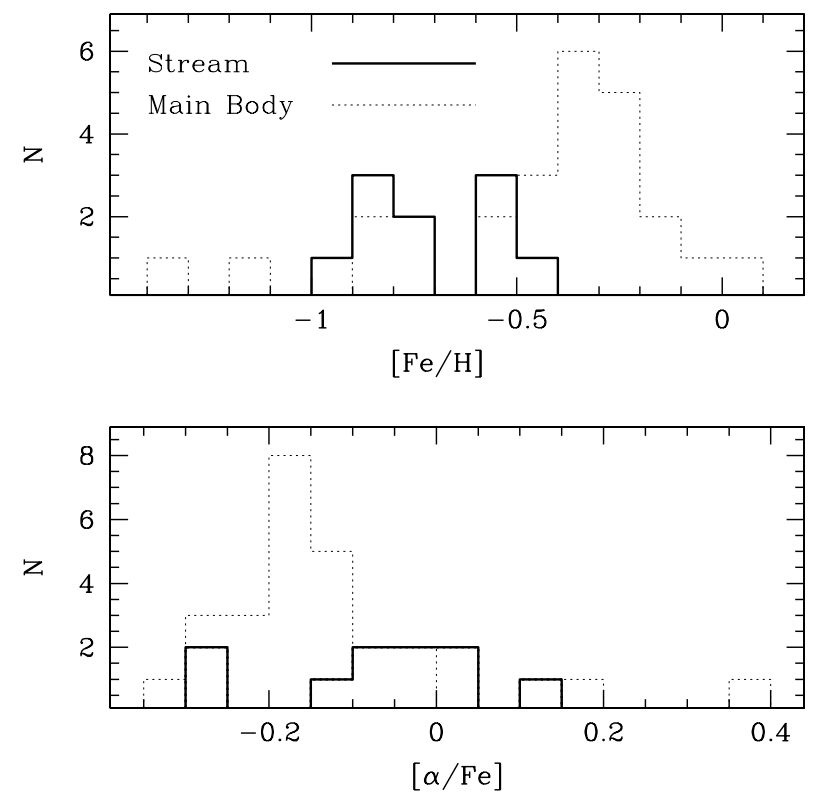

Fig. 8. Comparison between the metallicity (upper panel) and $[\alpha / \mathrm{Fe}]$ (lower panel) distribution of main body (dotted histogram) and stream (continuous histogram) stars.

The reader is referred to M04 for a discussion about the implications of the velocity dispersion in the Stream for the lumpiness of the galactic halo. We just recall here that a lumpy halo tends to heat coherent streams. However, the part of Stream we sample is populated by stars stripped in relatively recent times and, therefore, is probably not very sensitive to the lumpiness of the halo. We also presented $\mathrm{Fe}, \mathrm{Mg}$, and $\mathrm{Ca}$ abundances for 12 stars observed with the SARG facility. Ten of them are bona fide Sgr stream members as of their chemical abundances and RV (Figs. 7 and 3). Note, however, that any individual star can only be considered as a probable member.

In Fig. 8 (upper panel) we compare the Sgr main body (dotted histogram) and Stream (continuous histogram) metallicity distribution (MD). We point out that in M05, target stars were chosen in the infrared K vs. $(J-K)$ plane adopting the selection box of Fig. 1 in that paper. In the infrared plane, in fact, the upper Sgr RGB stands out very clearly from the contaminating MW field (to compare with the optical plane, see Fig. 2 in M05). Indeed, such a selection implies a bias toward metal rich stars, and, actually, we provided a thorough sampling of the Sgr dominant population (Monaco et al. 2002) at the Sgr center (i.e., around the globular cluster M 54, whose RGB is roughly represented by the bluer isochrone in Fig. 2 of M05).

The existence of a metal rich dominant population in Sgr allowed M03 to develop his successful technique for tracing the Sgr streams all over the sky. We used such a technique here to select our targets. It is easy, looking at Fig. 1 (and 2) in M03, to realize that the M03 and M05 selection criterion are practically the same. Note, that the mean temperature and gravity of the 15 stars analyzed in M05 (the first 15 lines of Table 1 in M05 ${ }^{10}$ ) are $3975 \mathrm{~K}$ and 1.00 (with $177 \mathrm{~K}$ and 0.18 as standard deviations, respectively) against the $3900 \mathrm{~K}$ and 0.9 adopted here for our stars.

B04 adopted a different selection function. Essentially, they selected fainter stars, which have slightly larger gravities. The abundances derived in M05 and B04 are compatible with each

\footnotetext{
10 Note that stars marked with an asterisk in Table 1 of M05 shows $\mathrm{TiO}$ bands and are not analyzed for chemical abundance there.
} 


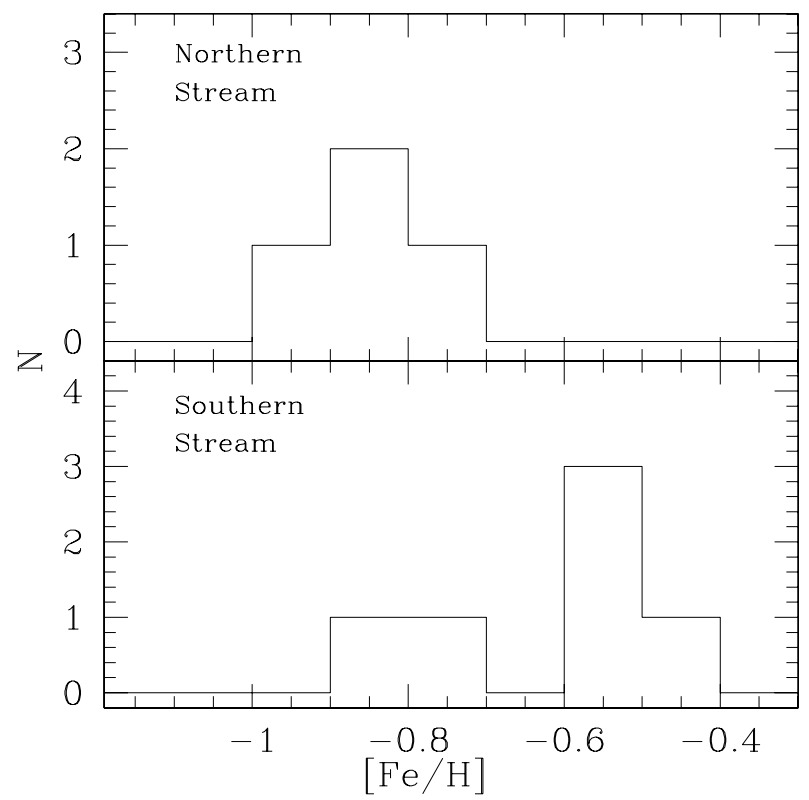

Fig. 9. Comparison between the metallicity distribution of stars in the Sgr trailing tail (lower panel) and in a portion of stream above (upper panel) the galactic plane. Stars in the upper panel sample a more ancient branch of the stream with respect to stars in the lower panel (see Fig. 1).

other within the errors (see M05 and Sbordone et al. 2006, for discussions).

Main body stars show a well defined peak in the MD at $\sim-0.35(\langle[\mathrm{Fe} / \mathrm{H}]\rangle=-0.35 \pm 0.19$, considering stars with $[\mathrm{Fe} / \mathrm{H}]>-0.80)$ and sample a large metallicity range. Stream stars sample a smaller metallicity range, as somewhat expected by the small number of stars analyzed. Clearly, the stream MD is shifted toward lower metallicity $(\langle[\mathrm{Fe} / \mathrm{H}]\rangle=-0.70 \pm 0.16)$ compared to the main body. A Kolmogorov-Smirnov test provides a probability $<10^{-3}$ that core and stream stars are extracted from the same parent population. A similar effect is also evident in the distribution of core and stream stars alpha element abundance ratio (lower panel).

SARG stars sample very different regions of the Stream (see Fig. 1). The sub-sample at negative galactic latitude (stars from \#1 to \#42; $b<0$ or $Y_{\mathrm{Sgr}, \mathrm{GC}}>0$ in Fig. 1) belongs to the Sgr trailing tail in the $80^{\circ}<\Lambda_{\odot}<100^{\circ}$ region. Hence, they were probably stripped during the last Sgr orbit. The $b>0$ sub-sample (stars from \#242 to \#283) traces, on the other hand, a more ancient episode of tidal stripping. In particular, according to the Law et al. (2005) model of Sgr disruption, they should have been lost three or more orbits ago (i.e., >2-3 Gyr ago). In Fig. 9 we plot the two sub-samples MD. It is evident that the mean metallicity of northern $(b>0)$ stream stars is lower compared to southern ones: $\langle[\mathrm{Fe} / \mathrm{H}]\rangle=-0.83 \pm 0.11$ and $\langle[\mathrm{Fe} / \mathrm{H}]\rangle=-0.61 \pm$ 0.13 , respectively. However, given the small number of stars in the two subsamples, this result has to be considered tentative and must be confirmed by the analysis of a more statistically significant sample of stars.

In summary, we found evidence of a more metal-poor MD in the Sgr Stream compared to the main body. Moreover, stars stripped in ancient orbits appear more metal-poor than stars lost in recent passages. Preliminary results pointing in the same direction were reported by Martínez-Delgado et al. (2004) and M03. Recently, more definitive indications in this sense were provided by Chou et al. (2006) (hereafter C06) and Bellazzini et al. (2006b).
In particular, $\mathrm{C} 06$ presented high resolution iron abundances for $56 \mathrm{M}$-giants belonging to the Sgr leading tail. Thus, we sample a different and complementary stream region. C06 found a variation of about -0.7 dex in the mean iron content from the core to the portion of stream they sample. Our results are qualitatively in agreement with C06, although the variation we find is a bit smaller. Several reasons can be responsible for such a difference (i.e., the different stream portion sampled, their more populated sample, the different analysis). C06 interpreted their results as a "direct evidence that there can be significant chemical differences between current dSph satellites and the bulk of the stars they have contributed to the halo". Our results confirm this statement. Moreover, as we pointed out in M05, Sgr stars at $[\mathrm{Fe} / \mathrm{H}]<$ -1 would also have $[\alpha / \mathrm{Fe}]$ abundance ratios similar to MW stars (see Fig. 7), hence they may be eligible as contributors to the assembly of the "normal" Galactic Halo (i.e., metal-poor and $\alpha$-enhanced). However, Sbordone et al. (2006) found under-solar or over-solar abundances for several elements in $12 \mathrm{Sgr}$ core stars. Even more interesting, they found a flat trend over the range $-0.9<[\mathrm{Fe} / \mathrm{H}]<0$ in some of the anomalous abundance ratios such as $[\mathrm{Na} / \mathrm{Fe}],[\mathrm{Zn} / \mathrm{Fe}]$, and $[\mathrm{Cu} / \mathrm{Fe}]$. Detailed abundances of Stream stars, especially of the most metal-poor ones, will be of the uttermost importance to finally establish whether Sgr stars lost in ancient passages could have significantly contributed to the standard stellar population of the galactic halo or not.

Moreover, the galactic halo is populated by very old stars. Under the assumption that dSph galaxies are dominated by an intermediate age population ("Carina-like"), Unavane et al. (1996) concluded that no more than $10 \%$ of the whole halo stellar population may have originated from accretion (but see Munoz et al. 2006). Indeed, these authors predicted low $[\alpha / \mathrm{Fe}]$ abundance ratios in the MW satellites as a result of their inferred low star formation rates (see Fig. 7).

The Sgr stellar content is dominated in the main body by an intermediate age (Bellazzini et al. 2006a) population. Stream stars studied here follow the abundance pattern of main body stars (Fig. 7) and are moderately more metal poor than them (Fig. 8). Hence, our targets likely have ages not much older than stars in the Sgr dominant population. Indeed, the SFH implied by the chemistry of stars sampled here, do not differ significantly from that of core stars: a prolonged period of star formation is needed to reach low $[\alpha / \mathrm{Fe}]$ abundance ratios. The C06 sample may eventually be made by older stars, more similar to the typical stellar content of the galactic halo.

However, neither our study nor the C06 one provide a fair sampling of the MD of the Sgr stream. We both selected targets using the 2MASS catalog. As such, the target selection biases our samples toward metal rich stars. As stated above, the target selection box was actually shaped to enclose stars in the Sgr dominant population. Thus, the actual MD of the Stream might eventually be skewed to even lower metallicities and made by older stars, for any reasonable age-metallicity relation (see, e.g., Layden \& Sarajedini 2000). Note also that Sgr is known to host a significant population of old and metal-poor stars (both in the main body and the streams, see Vivas et al. 2005; Monaco et al. 2003, and references therein).

Even SARG stars at $b>0$ could not have been stripped more then a few Gyr ago, a time at which the Sgr star formation was already completed. Therefore, we agree with the C06 conclusion that the difference in the core and stream MD witness a metallicity gradient inside the former Sgr (see, for instance, Bellazzini et al. 1999; Layden \& Sarajedini 2000, and references therein). Chemical abundances in the outskirts of the Sgr main body would be necessary to quantify metallicity gradients inside 
Sgr (see also Alard 2001). Stars in the trailing tail (lower panel in Fig. 9) are only mildly more metal poor than core ones (upper panel in Fig. 8). Hence, eventually, stars lying in the outer Sgr core and in the trailing tail might not present any chemical difference.

Indeed, the great majority of the MW satellites contain populations of old stars, either dominant or not. It appears a general tendency of the most metal-poor populations in dSphs to be less concentrated with respect to the other populations hosted (Munoz et al. 2006; Tolstoy et al. 2004, and references therein). This characteristic might favor the preferential stripping of metal-poor stars during tidal interactions between $\mathrm{dSphs}$ and the MW.

\section{Conclusions}

The Sgr SFH, its dynamical status and orbital evolution are constrained by the stellar populations hosted both in the main body and in the tidal streams of this disrupting galaxy. In this paper we presented radial velocities and chemical abundances $(\mathrm{Fe}, \mathrm{Mg}$, Ca) for a sample of stars belonging to the Sgr tidal streams. In particular, we presented the first $\alpha$-element abundances ever obtained for stars in the Sgr stream. The main results obtained can be summarized as follows:

- The velocity dispersion of the Sgr trailing tail $\left(8.3 \mathrm{~km} \mathrm{~s}^{-1}\right)$ is significantly lower than in the main body $\left(11.2 \mathrm{~km} \mathrm{~s}^{-1}\right)$.

- Stream stars follow the same distinct trend described by stars in the Sgr main body in the $[\mathrm{Fe} / \mathrm{H}]$ vs. $[\alpha / \mathrm{Fe}]$ plane (Fig. 7).

- Sgr stars are, on average, more metal-poor in the Stream than in the core (Fig. 8).

- Stars belonging to more ancient wraps of the Streams are more metal-poor (Fig. 9). This result was obtained comparing the MD of stars belonging to two different wraps of the Stream. However, given the limited number of stars in the two subsamples (4 and 6), this latter result has to be considered tentative.

Acknowledgements. We are grateful to A. Magazzù for his help in preparing the SARG observations. We also thank the La Silla SciOps department for their help in dealing with the HARPS spectra. Part of the data analysis has been performed using software developed by P. Montegriffo at the INAF - Osservatorio Astronomico di Bologna. The routines available at http://www. astro.virginia.edu/ srm $4 \mathrm{n} / \mathrm{Sgr} /$ were used to derive coordinates in the $\mathrm{Sgr}$ orbital plane. We acknowledge support from the MIUR/PRIN 2004025729, the INAF/PRIN05 CRA 1.06.08.02 and the INAF/PRIN05 CRA 1.06.08.03. PB also acknowledges support from EU contract MEXT-CT-2004-014265 (CIFIST).

\section{References}

Alard, C. 2001, A\&A, 377, 389

Alonso, A., Arribas, S., \& Martínez-Roger, C. 1999, A\&AS, 140, 261

Bean, J. L., Sneden, C., Hauschildt, P. H., Johns-Krull, C. M., \& Benedict, G. F. 2006, ApJ, in press [arXiv:astro-ph/0608093]

Bellazzini, M., Ferraro, F. R., \& Buonanno, R. 1999, MNRAS, 307, 619

Bellazzini, M., Correnti, M., Ferraro, F. R., Monaco, L., \& Montegriffo, P. 2006a, A\&A, 446, L1

Bellazzini, M., Newberg, H. J., Correnti, M., Ferraro, F. R., \& Monaco, L. 2006b, A\&A, 475, L21
Belokurov, V., et al. 2006a, ApJ Lett., submitted [arXiv: astro-ph/0605705]

Belokurov, V., Zucker, D. B., Evans, N. W., et al. 2006b, ApJ, 642, L137

Bertone, E., Buzzoni, A., Chavez, M., \& Rodriguez-Merino, L. H. 2004, AJ, 128,829

Bonifacio, P., Hill, V., Molaro, P., et al. 2000, A\&A, 359, 663

Bonifacio, P., Sbordone, L., Marconi, G., Pasquini, L., \& Hill, V. 2004, A\&A, 414, 503 (B04)

Chou, M.-Y., Majewski, S. R., Cunha, K., et al. 2006, ApJL, submitted [arXiv: astro-ph/0605101] (C06)

Duffau, S., Zinn, R., Vivas, A. K., et al. 2006, ApJ, 636, L97

Dohm-Palmer, R. C., Helmi, A., Morrison, H., et al. 2001, ApJ, 555, L37

Fuhr, J. R., \& Wiese, W. L. 1996, NIST Atomic Transition Probability Tables, CRC Handbook of Chemistry \& Physics, 77th edition, ed. D. R. Lide, CRC Press, Inc., Boca Raton, FL (NIST)

Fuhr, J. R., Martin, G. A., \& Wiese, W. L. 1988, J. Phys. Chem. Ref. Data, 17, Suppl. 4 (FMW)

Girardi, L., Bertelli, G., Bressan, A., et al. 2002, A\&A, 391, 195

Gratton, R. G., Carretta, E., Claudi, R., Lucatello, S., \& Barbieri, M. 2003, A\&A, 404, 187 (G03)

Hargreaves, J. C., Gilmore, G., \& Annan, J. D. 1996, MNRAS, 279, 108

Helmi, A., \& White, S. D. M. 1999, MNRAS, 307, 495

Ibata, R. A., Wyse, R. F. G., Gilmore, G., Irwin, M. J., \& Suntzeff, N. B. 1997, AJ, 113, 634

Ibata, R. A., Gilmore, G., \& Irwin, M. J. 1994, Nature, 370, 194

Kurucz, R. L. 1988, Trans. IAU, XXB, ed. M. McNally (Dordrecht: Kluwer), 168 (K88)

Kurucz, R. L. 1993a, CD-ROM 13, 18 http: //kurucz.harvard.edu

Kurucz, R. 1993b, Opacities for Stellar Atmospheres, Kurucz CD-ROM No. 2-12 Cambridge, Mass.: Smithsonian Astrophysical Observatory, http: //kurucz.harvard.edu/opacities.html

Kurucz, R. 1994, Atomic Data for Fe and Ni. Kurucz CD-ROM No. 22. Cambridge, Mass.: Smithsonian Astrophysical Observatory, 22 (K94)

Law, D. R., Johnston, K. V., \& Majewski, S. R. 2005, ApJ, 619, 807

Layden, A. C., \& Sarajedini, A. 2000, AJ, 119, 1760

Majewski, S. R., Skrutskie, M. F., Weinberg, M. D., \& Ostheimer, J. C. 2003, ApJ, 599, 1082 (M03)

Majewski, S. R., Kunkel, W. E., Law, D. R., et al. 2004, AJ, 128, 245 (M04)

Marconi, G., Buonanno, R., Castellani, M., et al. 1998, A\&A, 330, 453

Martin, G. A., Fuhr, J. R., \& Wiese, W. L. 1988. J. Phys. Chem. Ref. Data, 17, Suppl. 3 (MFW)

Martínez-Delgado, D., Gómez-Flechoso, M. Á., Aparicio, A., \& Carrera, R. 2004, ApJ, 601, 242

McWilliam, A., \& Smecker-Hane, T. A. 2005, ApJ, 622, L29

McWilliam, A., Rich, R. M., \& Smecker-Hane, T. A. 2003, ApJ, 592, L21

Monaco, L., Ferraro, F. R., Bellazzini, M., \& Pancino, E. 2002, ApJ, 578, L47

Monaco, L., Bellazzini, M., Ferraro, F. R., \& Pancino, E. 2003, ApJ, 597, L25

Monaco, L., Bellazzini, M., Ferraro, F. R., \& Pancino, E. 2004, MNRAS, 353, 874

Monaco, L., Bellazzini, M., Bonifacio, P., et al. 2005, A\&A, 441, 141 (M05)

Munoz, R. R., Majewski, S. R., Zaggia, S., et al. 2006, ApJ, 649, 201 (M06)

Newberg, H. J., Yanny, B., Rockosi, C., et al. 2002, ApJ, 569, 245

O'Brian, T. R., Wickliffe, M. E., Lawler, J. E., Whaling, J. W., \& Brault, W. 1991, Opt. Soc. Am. J. B Opt. Phys., 8, 1185 (O)

Olszewski, E. W., Pryor, C., \& Armandroff, T. E. 1996, AJ, 111, 750

Robertson, B., Bullock, J. S., Font, A. S., Johnston, K. V., \& Hernquist, L. 2005, ApJ, 632, 872

Sbordone, L., Bonifacio, P., Castelli, F., \& Kurucz, R. L. 2004, Mem. Soc. Astron. Ital. Suppl., 5, 93

Sbordone, L., Bonifacio, P., Buonanno, R., et al. 2006, A\&A, submitted

Schlegel, D. J., Finkbeiner, D. P., \& Davis, M. 1998, ApJ, 500, 525

Smith, G., \& Raggett, D. S. J. 1981, J. Phys. B At. Mol. Phys., 14, 4015 (SR)

Tolstoy, E., Irwin, M. J., Helmi, A., et al. 2004, ApJ, 617, L119

Unavane, M., Wyse, R. F. G., \& Gilmore, G. 1996, MNRAS, 278, 727

Valenti, J. A., Piskunov, N., \& Johns-Krull, C. M. 1998, ApJ, 498, 851

Venn, K. A., Irwin, M., Shetrone, M. D., et al. 2004, AJ, 128, 1177

Vivas, A. K., Zinn, R., \& Gallart, C. 2005, AJ, 129, 189

White, S. D. M., \& Rees, M. J. 1978, MNRAS, 183, 341

Wiese, W. L., Smith, M. W., \& Miles, B. M. 1969, NSRDS-NBS 22 (NBS) 


\section{Online Material}


L. Monaco et al.: RGB stars in the Sgr dSph streams. I., Online Material $p 2$

Table 1. Basic parameters of the program stars. Measured radial velocities and spectra signal-to-noise ratios are also reported. Beside the $2 \mathrm{MASS}$ name we give our own identifier to the star, which is used consequently in the other tables and throughout the paper.

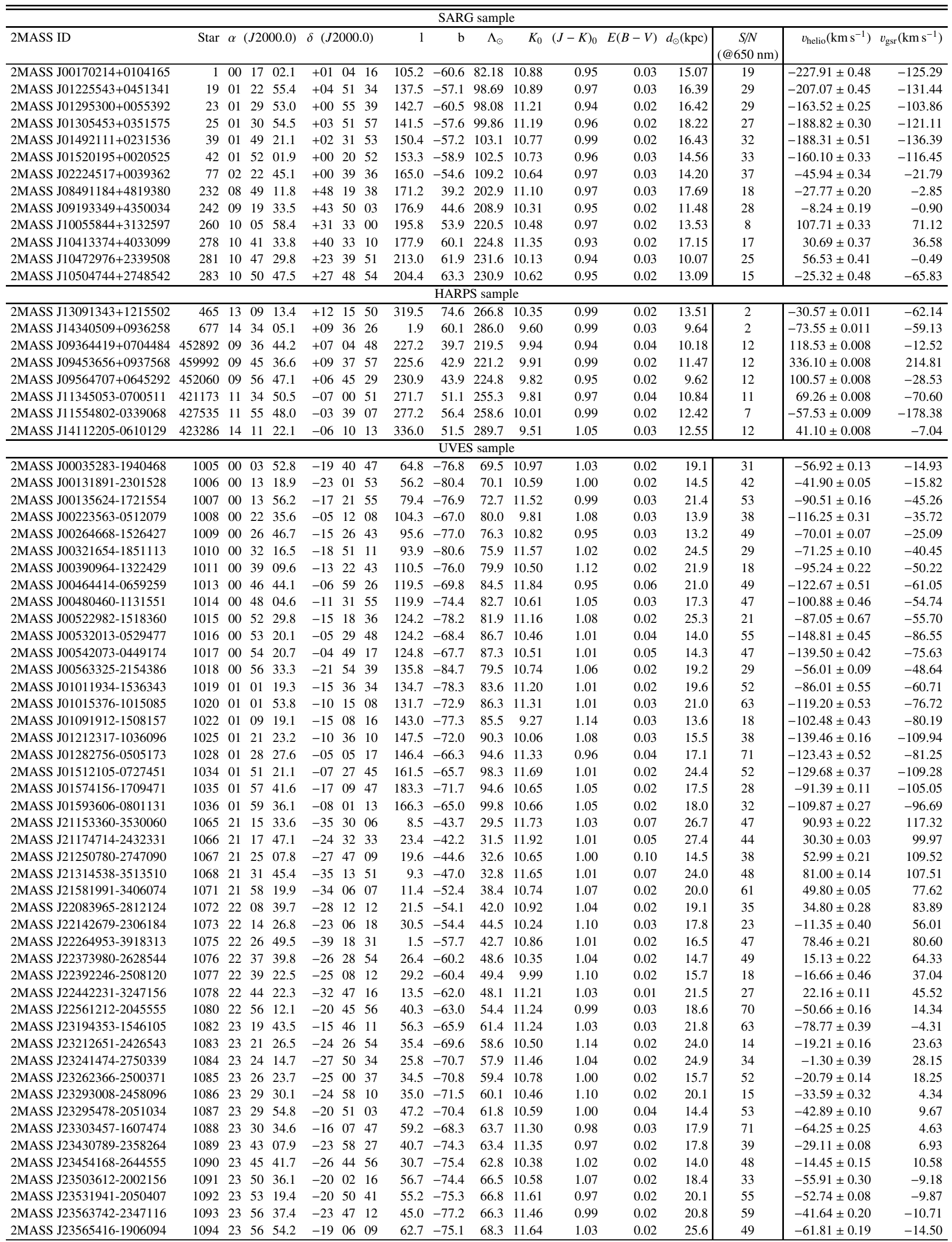


L. Monaco et al.: RGB stars in the Sgr dSph streams. I., Online Material p 3

\section{Appendix A: Individual line data}

The following tables report the line list and adopted atomic parameters for the program stars. The measured equivalent width and the corresponding abundance obtained for each line are also reported. 
L. Monaco et al.: RGB stars in the Sgr dSph streams. I., Online Material p 4

Table A.1. Line list and adopted atomic parameters for the program stars. The measured equivalent width and the corresponding abundance obtained for each line are also reported.

\begin{tabular}{|c|c|c|c|c|c|c|c|c|c|c|c|}
\hline Ion & $\begin{array}{r}\lambda \\
(\mathrm{nm})\end{array}$ & $\overline{\log g f}$ & $\begin{array}{l}\text { Source of } \\
\log g f \\
\text { (see notes) }\end{array}$ & $\begin{array}{r}E W \\
(\mathrm{pm}) \\
1\end{array}$ & $\epsilon$ & $\begin{array}{r}E W \\
(\mathrm{pm}) \\
19\end{array}$ & $\epsilon$ & $\begin{array}{r}E W(\mathrm{pm}) \\
(\mathrm{pm}) \\
23\end{array}$ & $\epsilon$ & $\begin{array}{r}E W \\
(\mathrm{pm}) \\
25\end{array}$ & $\epsilon$ \\
\hline Fe I & 585.5076 & -1.76 & FMW & - & - & - & - & 5.09 & 7.283 & - & - \\
\hline $\mathrm{Fe} \mathrm{I}$ & 588.3817 & -1.36 & FMW & - & - & 9.47 & 6.640 & 12.15 & 7.151 & 12.15 & 7.151 \\
\hline $\mathrm{Fe} \mathrm{I}$ & 595.2718 & -1.44 & FMW & 12.89 & 7.324 & 9.27 & 6.719 & 12.70 & 7.360 & 7.62 & 6.491 \\
\hline $\mathrm{Fe} \mathrm{I}$ & 602.7051 & -1.21 & FMW & 10.52 & 6.818 & 10.78 & 6.861 & 9.28 & 6.661 & 11.17 & 6.981 \\
\hline $\mathrm{Fe} \mathrm{I}$ & 605.6005 & -0.46 & FMW & 9.18 & 6.795 & 7.43 & 6.517 & 8.31 & 6.692 & 8.08 & 6.654 \\
\hline $\mathrm{Fe} \mathrm{I}$ & 609.6664 & -1.93 & FMW & - & - & 6.05 & 6.707 & 6.19 & 6.748 & 9.76 & 7.324 \\
\hline $\mathrm{Fe} \mathrm{I}$ & 615.1617 & -3.30 & FMW & 18.60 & 7.279 & 17.79 & 7.166 & 19.23 & 7.439 & 14.43 & 6.730 \\
\hline $\mathrm{Fe} \mathrm{I}$ & 622.6734 & -2.22 & FMW & - & - & 8.13 & 7.162 & - & - & - & - \\
\hline $\mathrm{Fe} \mathrm{I}$ & 651.8366 & -2.75 & FMW & 11.27 & 6.580 & 13.18 & 6.868 & 11.94 & 6.734 & 13.97 & 7.052 \\
\hline $\mathrm{Fe} \mathrm{I}$ & 659.7559 & -1.07 & FMW & 7.05 & 7.145 & 4.28 & 6.695 & 7.44 & 7.233 & 6.97 & 7.158 \\
\hline $\mathrm{Fe} \mathrm{I}$ & 670.3566 & -3.16 & FMW & 9.40 & 6.598 & 9.03 & 6.546 & 13.87 & 7.319 & - & - \\
\hline $\mathrm{Fe} \mathrm{I}$ & 673.9521 & -4.95 & FMW & 11.68 & 6.885 & 10.37 & 6.704 & 11.27 & 6.873 & 12.58 & 7.066 \\
\hline Fe I & 674.6954 & -4.35 & FMW & - & - & 4.74 & 6.923 & 6.99 & 7.254 & 5.55 & 7.052 \\
\hline $\mathrm{Fe} \mathrm{I}$ & 679.3258 & -2.47 & FMW & 3.75 & 6.989 & 3.12 & 6.872 & - & - & 5.08 & 7.224 \\
\hline $\mathrm{Fe} \mathrm{I}$ & 595.6694 & -4.60 & FMW & - & - & 20.24 & 7.026 & 20.59 & 7.225 & - & - \\
\hline $\mathrm{Fe} \mathrm{I}$ & 595.8333 & -4.42 & K94 & 9.36 & 7.040 & 10.36 & 7.187 & - & - & - & - \\
\hline $\mathrm{Fe} \mathrm{I}$ & 602.4058 & -0.12 & FMW & 17.44 & 7.486 & 14.06 & 6.988 & 11.71 & 6.661 & 13.83 & 7.016 \\
\hline $\mathrm{Fe} I$ & 606.5482 & -1.53 & FMW & 25.18 & 6.994 & 21.92 & 6.673 & 20.84 & 6.620 & 23.89 & 6.938 \\
\hline $\mathrm{Fe} \mathrm{I}$ & 624.6318 & -0.96 & FMW & 19.52 & 7.245 & 14.82 & 6.548 & 16.37 & 6.872 & 15.48 & 6.729 \\
\hline $\mathrm{Fe} \mathrm{I}$ & 625.2555 & -1.69 & FMW & - & - & 23.13 & 6.629 & 22.50 & 6.631 & 23.18 & 6.700 \\
\hline $\mathrm{Fe} I$ & 629.7793 & -2.74 & FMW & - & - & 18.29 & 6.714 & 19.64 & 6.967 & 17.54 & 6.685 \\
\hline $\mathrm{Fe} \mathrm{I}$ & 630.1500 & -0.67 & K94 & - & - & 14.88 & 6.335 & 15.81 & 6.560 & - & - \\
\hline $\mathrm{Fe} \mathrm{I}$ & 630.2494 & -1.13 & K94 & 12.39 & 6.442 & 13.55 & 6.629 & 17.73 & 7.360 & 16.04 & 7.106 \\
\hline $\mathrm{Fe} \mathrm{I}$ & 632.2685 & -2.43 & FMW & 15.38 & 6.551 & 14.90 & 6.476 & 17.76 & 7.001 & 17.16 & 6.909 \\
\hline $\mathrm{Fe} \mathrm{I}$ & 633.5330 & -2.23 & FMW & 25.32 & 6.923 & 22.29 & 6.643 & 22.59 & 6.733 & 21.80 & 6.654 \\
\hline $\mathrm{Fe} \mathrm{I}$ & 633.6823 & -1.05 & FMW & 19.20 & 7.397 & 16.53 & 7.016 & 16.27 & 7.054 & 15.30 & 6.900 \\
\hline $\mathrm{Fe} \mathrm{I}$ & 657.4227 & -5.04 & FMW & 18.29 & 7.107 & 17.54 & 6.990 & 17.25 & 7.037 & - & - \\
\hline $\mathrm{Fe} I$ & 659.3870 & -2.42 & FMW & 21.62 & 7.152 & 19.62 & 6.893 & 20.61 & 7.106 & 18.94 & 6.882 \\
\hline $\mathrm{Fe} I$ & 664.8080 & -5.29 & K94 & - & - & 11.18 & 6.325 & - & - & 12.35 & 6.538 \\
\hline $\mathrm{Fe} \mathrm{I}$ & 671.0318 & -4.88 & FMW & 11.55 & 6.686 & 13.05 & 6.900 & 10.81 & 6.627 & 12.96 & 6.946 \\
\hline $\mathrm{Fe} \mathrm{I}$ & 675.0152 & -2.62 & FMW & 20.26 & 7.233 & 17.74 & 6.829 & 18.33 & 7.024 & 18.84 & 7.109 \\
\hline $\mathrm{Fe} \mathrm{I}$ & 680.6843 & -3.21 & FMW & 10.46 & 6.744 & 11.22 & 6.853 & 13.74 & 7.287 & 13.32 & 7.222 \\
\hline $\mathrm{Fe} \mathrm{I}$ & 683.9830 & -3.45 & FMW & 12.10 & 6.963 & 11.26 & 6.842 & 14.43 & 7.374 & 12.47 & 7.071 \\
\hline Fe I & 722.3658 & -2.21 & $\mathrm{O}$ & 16.91 & 7.048 & - & - & 18.22 & 7.291 & 13.20 & 6.584 \\
\hline Fe I & 756.8899 & -0.87 & K94 & 13.63 & 7.114 & 11.48 & 6.809 & 12.45 & 7.000 & 13.39 & 7.136 \\
\hline $\mathrm{Fe} \mathrm{I}$ & 758.3788 & -1.99 & FMW & 15.44 & 6.590 & 17.23 & 6.836 & 16.66 & 6.829 & 19.54 & 7.208 \\
\hline $\mathrm{Fe} \mathrm{I}$ & 774.8269 & -1.76 & FMW & 21.39 & 6.989 & 17.32 & 6.488 & 19.73 & 6.868 & 19.91 & 6.890 \\
\hline $\mathrm{Fe} \mathrm{I}$ & 783.2196 & -0.02 & K94 & 15.37 & 6.719 & 14.80 & 6.641 & - & - & 16.70 & 6.962 \\
\hline Mg I & 552.8405 & -0.52 & G03 & 29.56 & 7.055 & 22.58 & 6.631 & 23.07 & 6.697 & 25.11 & 6.831 \\
\hline Mg I & 571.1088 & -1.73 & G03 & 13.92 & 6.848 & 11.55 & 6.519 & 14.39 & 6.956 & 15.21 & 7.069 \\
\hline $\mathrm{Mg} \mathrm{I}$ & 631.8717 & -1.94 & G03 & 5.87 & 7.037 & 4.83 & 6.884 & - & - & 9.15 & 7.521 \\
\hline $\mathrm{Mg} \mathrm{I}$ & 631.9237 & -2.16 & G03 & 6.78 & 7.386 & 5.26 & 7.169 & - & - & 5.28 & 7.183 \\
\hline Ca I & 585.7451 & 0.24 & SR & 18.52 & 5.373 & 17.00 & 5.171 & 19.85 & 5.596 & - & - \\
\hline $\mathrm{Ca} \mathrm{I}$ & 586.7562 & -1.49 & G03 & 6.07 & 5.356 & 6.59 & 5.424 & 6.59 & 5.439 & 7.31 & 5.535 \\
\hline $\mathrm{Ca} \mathrm{I}$ & 643.9075 & 0.39 & SR & - & - & 22.20 & 5.111 & 24.50 & 5.474 & 25.39 & 5.565 \\
\hline $\mathrm{Ca} \mathrm{I}$ & 645.5558 & -1.29 & SR & 14.51 & 5.654 & 12.81 & 5.403 & 13.25 & 5.523 & 12.34 & 5.385 \\
\hline $\mathrm{Ca} \mathrm{I}$ & 649.3781 & -0.11 & SR & 21.04 & 5.423 & 21.85 & 5.533 & 23.21 & 5.795 & 22.20 & 5.669 \\
\hline $\mathrm{Ca} I$ & 649.9650 & -0.82 & SR & 14.09 & 5.110 & 16.81 & 5.512 & - & - & 16.02 & 5.466 \\
\hline $\mathrm{Ca} I$ & 650.8850 & -2.11 & NBS & 6.57 & 5.402 & 4.86 & 5.180 & 6.27 & 5.377 & 5.66 & 5.297 \\
\hline $\mathrm{Ca}$ I & 714.8150 & 0.21 & K88 & 21.33 & 5.288 & 24.59 & 5.694 & 21.31 & 5.367 & 24.13 & 5.721 \\
\hline
\end{tabular}


L. Monaco et al.: RGB stars in the Sgr dSph streams. I., Online Material p 5

Table A.1. continued.

\begin{tabular}{|c|c|c|c|c|c|c|c|c|c|c|c|}
\hline Ion & $\begin{array}{r}\lambda \\
(\mathrm{nm})\end{array}$ & $\log g f$ & $\begin{array}{l}\text { Source of } \\
\log g f \\
\text { (see notes) }\end{array}$ & $\begin{array}{r}E W \\
\text { (pm) } \\
39 \\
\end{array}$ & $\epsilon$ & $\begin{array}{r}E W \\
(\mathrm{pm}) \\
42\end{array}$ & $\epsilon$ & $\begin{array}{r}E W(\mathrm{pm}) \\
(\mathrm{pm}) \\
77\end{array}$ & $\epsilon$ & $\begin{array}{r}E W \\
\text { (pm) } \\
232\end{array}$ & $\epsilon$ \\
\hline $\mathrm{Fe} \mathrm{I}$ & 585.5076 & -1.76 & FMW & - & - & - & - & 3.42 & 6.991 & & \\
\hline $\mathrm{Fe} \mathrm{I}$ & 588.3817 & -1.36 & FMW & 10.18 & 6.982 & 11.63 & 6.999 & 8.12 & 6.496 & - & - \\
\hline $\mathrm{Fe} \mathrm{I}$ & 595.2718 & -1.44 & FMW & 8.66 & 6.795 & - & - & 6.50 & 6.337 & 9.61 & 6.774 \\
\hline $\mathrm{Fe} \mathrm{I}$ & 602.7051 & -1.21 & FMW & 9.63 & 6.879 & 8.97 & 6.570 & 11.12 & 7.032 & 10.26 & 5.776 \\
\hline $\mathrm{Fe} \mathrm{I}$ & 605.6005 & -0.46 & FMW & 9.97 & 7.127 & 8.48 & 6.684 & 8.85 & 6.822 & - & - \\
\hline $\mathrm{Fe} \mathrm{I}$ & 609.6664 & -1.93 & FMW & - & - & 6.51 & 6.777 & - & - & - & - \\
\hline $\mathrm{Fe} \mathrm{I}$ & 615.1617 & -3.30 & FMW & 16.41 & 7.282 & 14.11 & 6.610 & 12.70 & 6.517 & - & - \\
\hline $\mathrm{Fe} \mathrm{I}$ & 622.6734 & -2.22 & FMW & 6.88 & 7.085 & 7.55 & 7.075 & 7.43 & 7.115 & - & - \\
\hline $\mathrm{Fe} \mathrm{I}$ & 651.8366 & -2.75 & FMW & 13.11 & 7.126 & 13.62 & 6.934 & 13.14 & 6.987 & - & - \\
\hline $\mathrm{Fe} \mathrm{I}$ & 659.7559 & -1.07 & FMW & 8.21 & 7.473 & 5.27 & 6.862 & 4.66 & 6.790 & 8.62 & 7.387 \\
\hline $\mathrm{Fe} \mathrm{I}$ & 670.3566 & -3.16 & FMW & 14.04 & 7.574 & 9.33 & 6.589 & 8.65 & 6.558 & 12.34 & 7.025 \\
\hline $\mathrm{Fe} \mathrm{I}$ & 673.9521 & -4.95 & FMW & 10.13 & 6.849 & 9.69 & 6.614 & 6.12 & 6.188 & 10.51 & 6.723 \\
\hline $\mathrm{Fe} \mathrm{I}$ & 674.6954 & -4.35 & FMW & 4.59 & 6.946 & 2.75 & 6.588 & - & - & 7.66 & 7.324 \\
\hline $\mathrm{Fe} \mathrm{I}$ & 679.3258 & -2.47 & FMW & 4.52 & 7.175 & - & - & 2.28 & 6.699 & 3.49 & 6.942 \\
\hline $\mathrm{Fe} \mathrm{I}$ & 595.6694 & -4.60 & FMW & - & - & - & - & - & - & 18.39 & 6.684 \\
\hline $\mathrm{Fe} \mathrm{I}$ & 595.8333 & -4.42 & K94 & - & - & 9.58 & 7.072 & 8.90 & 7.045 & - & - \\
\hline $\mathrm{Fe} \mathrm{I}$ & 602.4058 & -0.12 & FMW & 12.20 & 6.947 & 14.65 & 7.081 & 11.99 & 6.772 & - & - \\
\hline $\mathrm{Fe} \mathrm{I}$ & 606.5482 & -1.53 & FMW & 23.51 & 047 & 24.52 & 935 & 8.41 & 6.380 & - & - \\
\hline $\mathrm{Fe} \mathrm{I}$ & 624.6318 & -0.96 & FMW & 16.04 & 7.065 & 13.81 & 383 & 12.91 & 6.372 & 18.17 & 7.060 \\
\hline $\mathrm{Fe} \mathrm{I}$ & 625.2555 & -1.69 & FMW & 24.06 & 6.941 & 20.18 & 6.272 & 20.73 & 6.503 & - & - \\
\hline $\mathrm{Fe} \mathrm{I}$ & 629.7793 & -2.74 & FMW & 18.36 & 7.022 & 16.60 & 6.474 & 17.70 & 6.784 & 21.40 & 7.103 \\
\hline $\mathrm{Fe} \mathrm{I}$ & 630.1500 & -0.67 & K94 & 15.46 & 6.749 & - & - & - & - & 20.32 & 7.119 \\
\hline $\mathrm{Fe} \mathrm{I}$ & 630.2494 & -1.13 & K94 & 11.82 & 6.609 & 14.14 & 6.724 & 11.32 & 6.384 & 15.62 & 6.961 \\
\hline $\mathrm{Fe} \mathrm{I}$ & 632.2685 & -2.43 & FMW & 16.06 & 6.995 & 15.42 & 6.557 & 14.43 & 6.553 & 20.26 & 7.268 \\
\hline $\mathrm{Fe} \mathrm{I}$ & 633.5330 & -2.23 & FMW & 22.17 & 6.862 & 20.51 & 6.443 & 21.28 & 6.664 & 25.64 & 6.949 \\
\hline $\mathrm{Fe} \mathrm{I}$ & 633.6823 & -1.05 & FMW & 12.76 & 6.699 & 15.87 & 6.914 & 14.54 & 6.853 & 15.85 & 6.911 \\
\hline $\mathrm{Fe} \mathrm{I}$ & 657.4227 & -5.04 & FMW & 17.28 & 7.375 & 15.65 & 6.694 & 15.35 & 6.809 & 17.16 & 6.928 \\
\hline $\mathrm{Fe} \mathrm{I}$ & 659.3870 & -2.42 & FMW & 18.75 & 7.107 & 18.63 & 6.751 & 17.99 & 6.830 & 20.47 & 7.006 \\
\hline $\mathrm{Fe} \mathrm{I}$ & 54.8080 & -5.29 & K94 & - & - & - & - & - & - & - & - \\
\hline $\mathrm{Fe} \mathrm{I}$ & 1.0318 & -4.88 & MW & 12.46 & 7.067 & 11.02 & 6.614 & 9.63 & 6.499 & 13.63 & 6.985 \\
\hline $\mathrm{Fe} \mathrm{I}$ & 675.0152 & -2.62 & FMW & 7.57 & 7.223 & 17.65 & 314 & 15.25 & 6.596 & 17.93 & 6.860 \\
\hline $\mathrm{Fe} \mathrm{I}$ & 680.68 & -3.21 & FMW & 2.14 & 7.230 & 8.98 & 36 & 1.60 & 7.012 & 15.19 & .440 \\
\hline $\mathrm{Fe} \mathrm{I}$ & & -3.45 & FMW & 10.49 & 6.927 & 10.57 & 6.743 & .53 & 6.826 & 12.84 & 7.070 \\
\hline $\mathrm{Fe} \mathrm{I}$ & 722.3658 & -2.21 & $\mathrm{O}$ & 12.18 & 6.612 & 16.80 & 7.032 & 13.09 & 6.628 & - & - \\
\hline $\mathrm{Fe} \mathrm{I}$ & 756.8899 & -0.87 & K94 & 11.28 & 6.985 & 9.63 & 6.543 & 9.38 & 6.581 & 14.54 & 7.242 \\
\hline $\mathrm{Fe} \mathrm{I}$ & 758.3788 & -1.99 & FMW & 16.86 & 7.084 & 13.43 & 6.310 & 17.23 & 6.984 & 18.67 & 7.025 \\
\hline $\mathrm{Fe} \mathrm{I}$ & 774.8269 & -1.76 & FMW & 17.63 & 6.825 & 19.17 & 6.725 & - & - & 21.35 & 6.985 \\
\hline $\mathrm{Fe} \mathrm{I}$ & 783.2196 & -0.02 & K94 & 15.51 & 7.000 & 14.60 & 6.613 & 11.90 & 6.334 & 15.55 & 6.744 \\
\hline Mg I & 552.8405 & -0.52 & G03 & 21.73 & 6.681 & - & - & 25.85 & 6.904 & 35.63 & 7.285 \\
\hline Mg I & 571.1088 & -1.73 & G03 & 11.84 & 6.714 & 13.58 & 6.801 & 14.69 & 7.043 & 21.27 & 7.702 \\
\hline Mg I & 631.8717 & -1.94 & G03 & 5.25 & 6.993 & 5.78 & 7.024 & 5.58 & 7.019 & 8.01 & 7.337 \\
\hline Mg I & 631.9237 & -2.16 & G03 & - & - & 3.91 & 6.957 & 4.32 & 7.040 & 9.10 & 7.709 \\
\hline $\mathrm{Ca} I$ & 585.7451 & 0.24 & SR & - & - & 18.84 & 5.412 & - & - & - & - \\
\hline Ca I & 586.7562 & -1.49 & G03 & 6.30 & 5.452 & 6.57 & 5.421 & 6.05 & 5.380 & 9.29 & 5.778 \\
\hline $\mathrm{Ca} I$ & 643.9075 & 0.39 & SR & 23.35 & 5.560 & 26.09 & 5.566 & 24.89 & 5.582 & 28.62 & 5.791 \\
\hline $\mathrm{Ca} \mathrm{I}$ & 645.5558 & -1.29 & SR & 11.66 & 5.445 & 10.51 & 5.084 & 11.26 & 5.273 & 14.07 & 5.588 \\
\hline $\mathrm{Ca} \mathrm{I}$ & & -0.11 & SR & 21.88 & 5.869 & 18.99 & 5.127 & 24.52 & 6.011 & 29.15 & 6.299 \\
\hline $\mathrm{Ca} I$ & 649.9650 & -0.82 & SR & 15.63 & 5.644 & 18.92 & 5.829 & 16.29 & 5.587 & 19.82 & 5.961 \\
\hline $\mathrm{Ca} I$ & & -2.11 & NBS & 5.31 & 5.286 & 4.92 & 5.188 & 10.40 & 5.961 & 12.66 & 6.198 \\
\hline Ca I & 714.8150 & 0.21 & K88 & 21.63 & 5.668 & 24.18 & 5.646 & - & - & 27.45 & 5.991 \\
\hline
\end{tabular}


Table A.1. continued.

\begin{tabular}{|c|c|c|c|c|c|c|c|c|c|c|c|}
\hline Ion & $\begin{array}{r}\lambda \\
(\mathrm{nm})\end{array}$ & $\log g f$ & $\begin{array}{l}\text { source of } \\
\log g f \\
\text { (see notes) }\end{array}$ & $\begin{array}{r}E W \\
(\mathrm{pm}) \\
242\end{array}$ & $\epsilon$ & $\begin{array}{r}E W \\
(\mathrm{pm}) \\
278\end{array}$ & $\epsilon$ & $\begin{array}{r}E W(\mathrm{pm}) \\
(\mathrm{pm}) \\
281\end{array}$ & $\epsilon$ & $\begin{array}{r}E W \\
(\mathrm{pm}) \\
283\end{array}$ & $\epsilon$ \\
\hline $\mathrm{I}$ & 585.5076 & -1.76 & FMW & 5.72 & 7.352 & - & - & - & - & - & - \\
\hline $\mathrm{Fe} \mathrm{I}$ & 588.3817 & -1.36 & FMW & 11.53 & 6.927 & 9.43 & 6.677 & - & _ & - & _ \\
\hline $\mathrm{Fe} \mathrm{I}$ & 595.2718 & -1.44 & FMW & - & - & - & - & 7.76 & 6.455 & 7.05 & 6.400 \\
\hline $\mathrm{Fe} \mathrm{I}$ & 602.7051 & -1.21 & FMW & 10.49 & 6.768 & - & - & - & - & 10.04 & 6.787 \\
\hline $\mathrm{Fe} \mathrm{I}$ & 605.6005 & -0.46 & FMW & 10.37 & 6.943 & - & - & - & - & 9.90 & 6.956 \\
\hline $\mathrm{Fe} \mathrm{I}$ & 609.6664 & -1.93 & FMW & 7.12 & 6.846 & - & - & 4.76 & 6.494 & - & - \\
\hline $\mathrm{Fe} \mathrm{I}$ & 615.1617 & -3.30 & FMW & - & - & - & - & - & - & - & - \\
\hline $\mathrm{Fe} \mathrm{I}$ & 622.6734 & -2.22 & FMW & - & - & - & - & - & - & - & - \\
\hline $\mathrm{Fe} \mathrm{I}$ & 651.8366 & -2.75 & FMW & 14.65 & 7.025 & 12.76 & 6.863 & 9.72 & 6.320 & - & - \\
\hline $\mathrm{Fe} \mathrm{I}$ & 659.7559 & -1.07 & FMW & 5.24 & 6.842 & - & - & - & - & - & - \\
\hline $\mathrm{Fe} \mathrm{I}$ & 670.3566 & -3.16 & FMW & 12.66 & 7.019 & 11.00 & 6.873 & 10.05 & 6.655 & 11.96 & 7.021 \\
\hline $\mathrm{Fe} \mathrm{I}$ & 673.9521 & -4.95 & FMW & 10.45 & 6.679 & 9.00 & 6.553 & 7.72 & 6.342 & 13.07 & 7.141 \\
\hline $\mathrm{Fe} \mathrm{I}$ & 674.6954 & -4.35 & FMW & - & - & - & - & 5.22 & 6.981 & - & - \\
\hline $\mathrm{Fe} \mathrm{I}$ & 679.3258 & -2.47 & FMW & 4.91 & 7.170 & - & - & - & - & 3.55 & 6.962 \\
\hline $\mathrm{Fe} I$ & 595.6694 & -4.60 & FMW & 16.41 & 6.242 & - & - & 19.00 & 6.683 & 19.00 & 6.919 \\
\hline $\mathrm{Fe} \mathrm{I}$ & 595.8333 & -4.42 & K94 & 7.40 & 6.747 & - & - & 8.38 & 6.876 & 6.24 & 6.627 \\
\hline $\mathrm{Fe} \mathrm{I}$ & 602.4058 & -0.12 & FMW & 12.83 & 6.728 & - & - & 12.05 & 6.603 & 10.68 & 6.486 \\
\hline $\mathrm{Fe} \mathrm{I}$ & 606.5482 & -1.53 & FMW & - & - & 23.45 & 6.895 & 21.45 & 6.542 & 21.61 & 6.708 \\
\hline $\mathrm{Fe} \mathrm{I}$ & 624.6318 & -0.96 & FMW & 15.55 & 6.589 & - & - & 15.87 & 6.639 & - & - \\
\hline $\mathrm{Fe} \mathrm{I}$ & 625.2555 & -1.69 & FMW & - & - & 26.25 & 6.971 & - & - & 24.47 & 6.823 \\
\hline $\mathrm{Fe} \mathrm{I}$ & 629.7793 & -2.74 & FMW & 15.56 & 6.248 & 21.39 & 7.168 & - & - & 15.42 & 6.369 \\
\hline $\mathrm{Fe} \mathrm{I}$ & 630.1500 & -0.67 & K94 & 16.14 & 6.459 & 15.86 & 6.568 & 17.10 & 6.605 & 15.84 & 6.565 \\
\hline $\mathrm{Fe} \mathrm{I}$ & 630.2494 & -1.13 & K94 & 13.02 & 6.483 & 12.69 & 6.553 & 12.64 & 6.425 & 13.47 & 6.684 \\
\hline $\mathrm{Fe} \mathrm{I}$ & 632.2685 & -2.43 & FMW & 15.12 & 6.441 & 15.88 & 6.708 & 15.14 & 6.444 & 15.07 & 6.578 \\
\hline $\mathrm{Fe} \mathrm{I}$ & 633.5330 & -2.23 & FMW & - & - & 24.91 & 6.940 & 20.67 & 6.391 & 22.60 & 6.734 \\
\hline $\mathrm{Fe} \mathrm{I}$ & 633.6823 & -1.05 & FMW & 13.71 & 6.505 & - & - & 13.92 & 6.540 & 12.88 & 6.501 \\
\hline $\mathrm{Fe} \mathrm{I}$ & 657.4227 & -5.04 & FMW & 14.60 & 6.471 & 18.63 & 7.268 & 16.10 & 6.687 & 15.88 & 6.810 \\
\hline $\mathrm{Fe} \mathrm{I}$ & 659.3870 & -2.42 & FMW & 17.24 & 6.469 & 19.73 & 6.990 & 17.47 & 6.503 & 17.39 & 6.655 \\
\hline $\mathrm{Fe} \mathrm{I}$ & 664.8080 & -5.29 & K94 & - & - & 11.28 & 6.383 & - & - & 12.07 & 6.497 \\
\hline $\mathrm{Fe} \mathrm{I}$ & 671.0318 & -4.88 & FMW & 9.44 & 6.374 & 9.86 & 6.493 & 8.40 & 6.246 & 11.99 & 6.799 \\
\hline $\mathrm{Fe} \mathrm{I}$ & 675.0152 & -2.62 & FMW & 14.22 & 6.206 & 16.74 & 6.758 & 16.87 & 6.605 & 15.44 & 6.541 \\
\hline $\mathrm{Fe} \mathrm{I}$ & 680.6843 & -3.21 & FMW & 11.65 & 6.869 & - & - & 10.22 & 6.673 & 11.97 & 7.014 \\
\hline $\mathrm{Fe} \mathrm{I}$ & 683.9830 & -3.45 & FMW & - & - & - & - & - & - & - & - \\
\hline $\mathrm{Fe} \mathrm{I}$ & 722.3658 & -2.21 & $\mathrm{O}$ & - & - & - & - & 11.75 & 6.278 & 12.76 & 6.520 \\
\hline $\mathrm{Fe} \mathrm{I}$ & 756.8899 & -0.87 & K94 & 10.44 & 6.620 & - & - & 8.56 & 6.361 & 10.48 & 6.707 \\
\hline $\mathrm{Fe} \mathrm{I}$ & 758.3788 & -1.99 & FMW & 16.06 & 6.611 & - & - & 14.70 & 6.429 & - & - \\
\hline $\mathrm{Fe} I$ & 774.8269 & -1.76 & FMW & 16.95 & 6.370 & 20.60 & 6.971 & 19.16 & 6.652 & - & - \\
\hline $\mathrm{Fe} \mathrm{I}$ & 783.2196 & -0.02 & K94 & - & - & - & - & - & - & 15.40 & 6.786 \\
\hline Mg I & 552.8405 & -0.52 & G03 & 27.28 & 6.913 & 23.13 & 6.701 & 24.86 & 6.765 & 23.99 & 6.759 \\
\hline Mg I & 571.1088 & -1.73 & G03 & 16.77 & 7.177 & - & - & 15.06 & 6.958 & 13.41 & 6.819 \\
\hline Mg I & 631.8717 & -1.94 & G03 & 6.01 & 7.045 & 5.10 & 6.935 & 2.93 & 6.554 & 6.12 & 7.086 \\
\hline Mg I & 631.9237 & -2.16 & G03 & - & - & 4.42 & 7.049 & 4.60 & 7.060 & 4.61 & 7.079 \\
\hline $\mathrm{Ca} I$ & 585.7451 & 0.24 & SR & - & - & - & - & - & - & - & - \\
\hline $\mathrm{Ca} \mathrm{I}$ & 586.7562 & -1.49 & G03 & 8.14 & 5.603 & 6.08 & 5.370 & 8.39 & 5.658 & 9.15 & 5.788 \\
\hline $\mathrm{Ca} \mathrm{I}$ & 612.2217 & -0.31 & NIST & - & - & - & - & - & - & 26.64 & 5.527 \\
\hline $\mathrm{Ca} \mathrm{I}$ & 643.9075 & 0.39 & SR & 25.02 & 5.378 & 24.47 & 5.471 & 27.48 & 5.693 & 22.89 & 5.286 \\
\hline $\mathrm{Ca} \mathrm{I}$ & 645.5558 & -1.29 & SR & 13.46 & 5.445 & 11.82 & 5.310 & 15.41 & 5.788 & 10.51 & 5.120 \\
\hline $\mathrm{Ca} \mathrm{I}$ & 649.3781 & -0.11 & SR & 21.55 & 5.408 & 22.55 & 5.713 & 21.13 & 5.436 & - & - \\
\hline $\mathrm{Ca} \mathrm{I}$ & 649.9650 & -0.82 & SR & 15.81 & 5.297 & 17.32 & 5.667 & 14.56 & 5.179 & - & - \\
\hline $\mathrm{Ca} \mathrm{I}$ & 650.8850 & -2.11 & NBS & 7.85 & 5.542 & 7.30 & 5.510 & 9.29 & 5.742 & 5.03 & 5.212 \\
\hline $\mathrm{Ca} \mathrm{I}$ & 714.8150 & 0.21 & K88 & 25.94 & 5.763 & 24.47 & 5.761 & 22.89 & 5.486 & 22.59 & 5.535 \\
\hline
\end{tabular}

FMW - Fuhr et al. (1988);

NIST - Fuhr \& Wiese (1996)

G03 - Gratton et al. (2003);

SR - Smith et al. (1981);

NBS - Wiese et al. (1969);

MFW - Martin et al. (1988);

O - O'Brian et al. (1991);

K88 - Kurucz (1988);

K94 - Kurucz (1994). 